JOURNAL OF ETHNOBIOLOGY

AND ETHNOMEDICINE

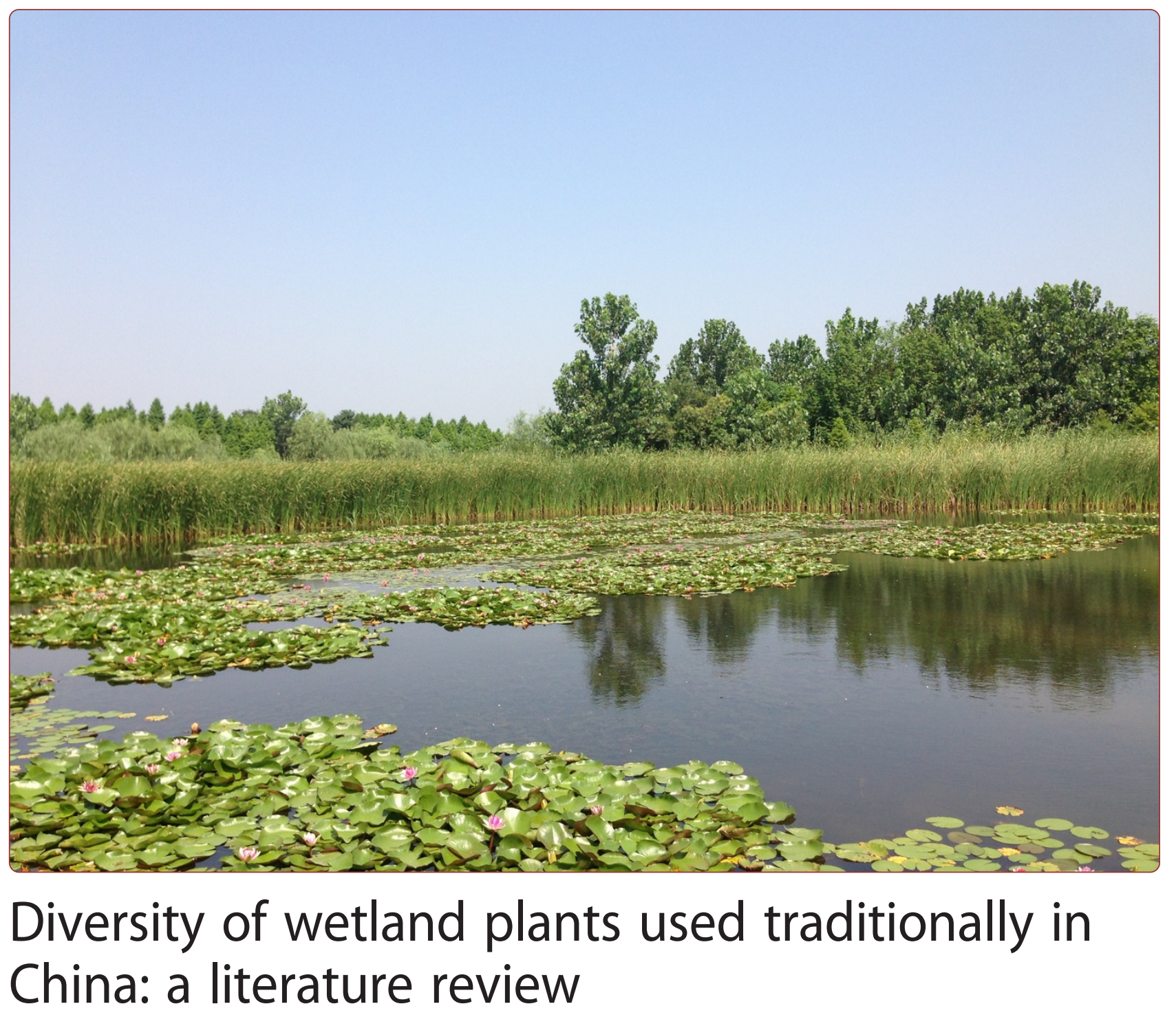

Zhang et al. 


\title{
Diversity of wetland plants used traditionally in China: a literature review
}

\author{
Yin Zhang, Hualin Xu, Hui Chen, Fei Wang and Huyin Huai*
}

\begin{abstract}
Background: In comparison with terrestrial plants, those growing in wetlands have been rarely studied ethnobotanically, including in China, yet people living in or near wetlands can accumulate much knowledge of the uses of local wetland plants. A characteristic of wetlands, cutting across climatic zones, is that many species are widely distributed, providing opportunities for studying general patterns of knowledge of the uses of plants across extensive areas, in the present case China. There is urgency in undertaking such studies, given the rapid rates of loss of traditional knowledge of wetland plants as is now occurring.

Methods: There have been very few studies specifically on the traditional knowledge of wetland plants in China. However, much information on such knowledge does exist, but dispersed through a wide body of literature that is not specifically ethnobotanical, such as regional Floras. We have undertaken an extensive study of such literature to determine which species of wetland plants have been used traditionally and the main factors influencing patterns shown by such knowledge. Quantitative techniques have been used to evaluate the relative usefulness of different types of wetland plants and regression analyses to determine the extent to which different quantitative indices give similar results.
\end{abstract}

Results: 350 wetland plant species, belonging to 66 families and 187 genera, were found to have been used traditionally in China for a wide range of purposes. The top ten families used, in terms of numbers of species, were Poaceae, Polygonaceae, Cyperaceae, Lamiaceae, Asteraceae, Ranunculaceae, Hydrocharitaceae, Potamogetonaceae, Fabaceae, and Brassicaceae, in total accounting for $58.6 \%$ of all species used. These families often dominate wetland vegetation in China. The three most widely used genera were Polygonum, Potamogeton and Cyperus. The main uses of wetlands plants, in terms of numbers of species, were for medicine, food, and forage. Three different ways of assigning an importance value to species (Relative Frequency of Citation RFC; Cultural Importance Cl; Cultural Value Index (V) all gave similar results.

Conclusions: A diverse range of wetland plants, in terms of both taxonomic affiliation and type of use, have been used traditionally in China. Medicine, forage and food are the three most important categories of use, the plants providing basic resources used by local people in their everyday lives. Local availability is the main factor influencing which species are used. Quantitative indexes, especially Cultural Value Index, proved very useful for evaluating the usefulness of plants as recorded in the literature.

Keywords: Wetland plants, Traditional knowledge, Literature study, China

\footnotetext{
*Correspondence: hyhuai@yzu.edu.cn

College of Bioscience and Biotechnology, Yangzhou University, Yangzhou
} 225009, China 


\section{Background}

Traditional knowledge of plants has played an important role in people's lives historically and has the potential to continue to contribute much in the future for the sustainable development of societies and economies [1-3]. However, as with biodiversity, traditional knowledge is becoming endangered with the danger of being total loss $[4,5]$. There are many causes of such endangerment, including changes occurring in the environment [6], urbanization and economic globalization $[7,8]$. Urbanization is one of the most important factors globally causing loss of traditional knowledge $[7,8]$.

In contrast with terrestrial ecosystems, wetlands have been poorly studied ethnobotanically, even though, for people living in and around wetlands, wild wetland plants play important roles in their daily lives [9-12]. Plants are collected from wetlands for a wide variety of purposes, such as provision of medicine, food and building materials and to sell for cash income $[9,11,13]$. Wetlands are very susceptible to loss or degradation through urbanization [14-16], which can change their extent and species composition and lead to the loss of biodiversity [17-19]. Traditional knowledge about wetlands is declining along with wetland degradation and alteration [11,12], an inevitable trend given the accelerating rate of urbanization that is now occurring.

Wetlands are widely distributed throughout China, but especially common in the east and south [20], where there are particularly rich traditions of local knowledge about the uses of their plants. There has been little ethnobotanical research specifically on wetland plants, but much information on traditional uses of wetland plants nevertheless does exist, though scattered through regional floras and other types of publication. Like traditional ethnobotanical knowledge generally, that concerned with wetland plants is becoming [11]. Ethnobotanical research on people's knowledge of wetland plants in China is urgently needed.

Currently, most ethnobotanical research concerned with any habitat type (not just wetlands) is conducted on the basis of case studies undertaken at specific field locations. The results of such studies are important for understanding relationships between local people and their environments, including sometimes for providing guidance on the sustainable use of plants and their conservation. However, case studies unavoidably emphasize unique local features of the relationships between people and their environments [21]. There is a role for systematic reviews and meta-analyses on wider regional to international scales to investigate general patterns of knowledge and use relating to plants, including to provide contexts for local-level studies [21-25].

Quantitative methods have been successfully applied in ethnobotanical studies, especially in the evaluation of cultural value or importance of species $[11,24,26]$. However, most quantitative methods have been developed for the analysis of case studies based on field work. Which of these methods is most suitable for systematic reviews or meta-analyses remains little studied, with little published information available.

In this paper, we aim to answer the following questions through a study of the literature: 1 . What are the botanical characteristics of wetland plants traditionally considered useful in China? 2. What are the main factors influencing the patterns of use of wetland plants in China? 3. Which of the available quantitative indexes is most suitable for evaluating traditional knowledge, as determined from the literature?

\section{Methods}

\section{Data collection}

Two criteria were used to identify the species included in this analysis. First, the species had to be wetland plants; we took the definition of a wetland as that given in the Convention on Wetlands of International Importance especially as Waterfowl Habitat (1971). Second, the species had to have been recorded as having traditional use. In this paper, we only paid attention on vascular plants. There have been very few systematic ethnobotanical studies conducted on wetlands in China. Most of the available ethnobotanical information on wetland plants is scattered sporadically through various publications, such as national and provincial floras, economic floras, and papers published in scientific journals. Our approach has been to identify, so far as we were able, all sources of potential information on wetland plants and then to search through this literature to compile an ethnobotanical inventory of wetland plants. Then we used the scientific names of the plants as key words to search further information on traditional use in the China Science and Technology Journal Database. The total number of principal literature sources studied was 56 [27-82].

Based on records in the literature, we classified uses into 11 groups: medicine, fodder, food, green manure, fiber, ornamental, liquor-making, environmental, industrial raw material, pesticide, and other. The medicine category includes plants used for treating animal as well as human diseases. Fodder refers to plants eaten by domestic animals. Edible plants are those as human food either in a raw or processed state. Green manure refers to plants employed as fertilizer. Fiber plants are those yielding fibers used by people; there are various ways in which they are extracted from the plants. Ornamental plants are those planted deliberately to beautify the environment. Liquor-making plants are those yielding either basic ingredients or supplementary materials used in making traditional liquor. The environmental category refers to plants used in soil conservation or the stabilization of dams. The industrial raw material category includes those plants providing raw materials for industrial production, such as for the manufacture of 
essential oils. Although not a typical traditional use, such plants can provide local people with sources of cash income and thus is important category of use for some people. The pesticide category refers to plants used for killing or driving away pests such as insects. Uses other than those in the above ten categories are grouped together in 'other'.

\section{Data analysis}

Use Value (UV) is a widely used statistic employed by ethnobotanists to provide a measure of the relative usefulness of plants to people $[24,83]$. In this paper, we use the formula $U V_{i}=\Sigma U_{i} / n$ to calculate the use value of each species (i), $U_{i}$ referring to the number of categories of use mentioned for a species in a particular literature source and $\mathrm{n}$ the total number of literature sources mentioning the species $[23,24,84]$. For example, if two literature sources $(n=2)$ mention species $i$, with three use categories mentioned in the first source and one in the second, then $\mathrm{UV}_{\mathrm{i}}=(3+1) / 2=2$.

Family Use Value (FUV), a statistic developed by Phillips and Gentry [83], provides a measure of the relative usefulness of plant families. FUV for a particular family (j) is calculated using the formula $\mathrm{FUV}_{\mathrm{j}}=\Sigma\left(\mathrm{UV}_{\mathrm{i}}\right) / \mathrm{n}$, where $\mathrm{UV}_{\mathrm{i}}$ is the use value of species $i$ and $n$ is the number of species in the family.

The statistic Relative Frequency of Citation $\left(\mathrm{RFC}_{\mathrm{i}}\right)$ is used as a measure of consensus between the information provided by different literature sources. RFC is similar conceptually to that of Utilization Frequency proposed by Ladio and Lozada [85]. RFC for a species is calculated as $\mathrm{RFC}_{\mathrm{i}}=\mathrm{FC}_{\mathrm{i}} / \mathrm{N}$ [24], where $\mathrm{FC}_{\mathrm{i}}$ is the number of literature sources mentioning species $\mathrm{i}$ and $\mathrm{N}$ the total number of literature sources consulted ( $\mathrm{N}=56$ in the present case).

The cultural value (or importance value) of species in a given culture and the comparative importance of species interculturally are receiving growing attention in ethnobotanical studies, especially those concerned with medicinal plants $[24,25,86,87]$. Here, measures of cultural value for wetland plant in China are provided by the statistics Cultural Importance Index (CI) and Cultural Value Index $(\mathrm{CV})$, based on formulae given in Tardio \& Pardo-deSantayana and Reyes-Garcia et al. [24,26].

Finally, regression analysis has been used to determine the relationships between $\mathrm{RFC}, \mathrm{CI}$ and $\mathrm{CV}$.

\section{Results}

\section{Diversity of the useful wetland plants in China}

A total of 350 wetland plant species (including 5 varieties), belonging to 66 families and 187 genera, were recorded as used in China according to the survey. The average number of species recorded per family was 5.3, with 15 families (22.7\% of the total) having more species than the average (Table 1). The ten families (Poaceae, Polygonaceae, Cyperaceae, Lamiaceae, Asteraceae, Ranunculaceae,
Hydrocharitaceae, Potamogetonaceae, Fabaceae, and Brassicaceae) contributed $58.6 \%$ of all species, the 5 with the highest number of species being Poaceae (46 species; $13.1 \%$ of the total), Polygonaceae (9.1\%), Cyperaceae (8.3\%), Lamiaceae (5.7\%), and Asteraceae (5.1\%), Twenty-five families (37.9\% of the total) were represented by only one useful species each. The remaining 33 families contributed between 2 and 11 species each (0.6-3.1\% of the total).

Some taxa were obviously dominant at the generic level, 32 genera (17.1\% of the total) being represented by 3 or more species. The top scorer was Polygonum (24 species), followed by: Potamogeton (12); Cyperus (10); Scirpus and Rumex (both 7); Ranunculus (6); Carex, Eriocaulon, Echinochloa, Cardamine, and Potentilla (all 5); Blyxa, Bromus, Eleocharis, Equisetum, Lysimachia, Najas, Paspalum, Stachys, Trapa, and Typha (all 4); and then Alisma, Arisaema, Clematis, Leersia, Ludwigia, Miscanthus, Monochoria, Murdannia, Oenanthe, Plantago, and Rorippa (all 3). The dominant genera belonged to the same families as scored highest at the family level, for example Polygonaceae, Cyperaceae, Potamogetonaceae, Poaceae, Hydrocharitaceae and Ranunculaceae.

Scores for Family Use Value (FUV) fell between 1 (for 14 families) and 3.2 (Cucurbitaceae) (Table 1). The top 10 families according to this measure (all with FUV >2.0) were completely different from those scoring highly according to number of species. There was no obvious correlation between FUV and number of species used per family. All top 10 families based on FUV were families with few wetland species ( 3 or fewer). However, there were also families having few species with low FUV scores.

\section{Characteristics of traditional use of wetland plants}

Medicine, fodder and food were the main uses made of wetland plants according to number of species (Table 2). Seventy percent of all species were recorded to be of medicinal use, nearly half of were employed as forage and somewhat fewer as food. Fewer plants were recorded as employed for green manure, fiber, or as sources of raw materials for industry, but all these were noticeably important types of use. The other five categories of use accounted for only a small proportion of total uses. Twenty-six species (7.4\% of all species) provided insecticides and 22 species (6.3\%) were employed in the making of liquor. Several plants were sold for cash, such as species of Polygonum, among others. The 'Other' category included some plants used for skin care, such as Coix lacryma-jobi and Zizania latifolia, and others in house construction, such as Arundo donax, Miscanthus sacchariflorus, and Phragmites australis. Although few species were included in the construction category, nevertheless wetland plants used in construction can be of major importance to local people. 
Table 1 The taxonomic composition of wetland plants used traditionally and family use values (FUV) based on literature research

\begin{tabular}{|c|c|c|c|}
\hline Family & No. of genus (\%) & No. of Species (\%) & FUV \\
\hline Poaceae & $27(14.4)$ & $46(13.1)$ & 1.59 \\
\hline Polygonaceae & $3(1.6)$ & $32(9.1)$ & 1.57 \\
\hline Cyperaceae & $6(3.2)$ & $29(8.3)$ & 1.34 \\
\hline Lamiaceae & $14(7.5)$ & $20(5.7)$ & 1.25 \\
\hline Asteraceae & $14(7.5)$ & $18(5.1)$ & 1.30 \\
\hline Ranunculaceae & $6(3.2)$ & $14(4.0)$ & 1.10 \\
\hline Hydrocharitaceae & $6(3.2)$ & $13(3.7)$ & 1.51 \\
\hline Potamogetonaceae & $1(0.5)$ & $12(3.4)$ & 1.33 \\
\hline Fabaceae & $11(5.9)$ & $11(3.1)$ & 1.97 \\
\hline Brassicaceae & $4(2.1)$ & $10(2.9)$ & 1.81 \\
\hline Apiaceae & $5(2.7)$ & $7(2.0)$ & 1.38 \\
\hline Araceae & $5(2.7)$ & $7(2.0)$ & 1.19 \\
\hline Rosaceae & $3(1.6)$ & $7(2.0)$ & 1.56 \\
\hline Scrophulariaceae & $6(3.2)$ & $7(2.0)$ & 1.05 \\
\hline Alismataceae & $3(1.6)$ & $6(1.7)$ & 1.29 \\
\hline Chenopodiaceae & $3(1.6)$ & $5(1.4)$ & 1.86 \\
\hline Commelinaceae & $2(1.1)$ & $5(1.4)$ & 1.24 \\
\hline Eriocaulaceae & $1(0.5)$ & $5(1.4)$ & 1.00 \\
\hline Primulaceae & $2(1.1)$ & $5(1.4)$ & 1.24 \\
\hline Typhaceae & $2(1.1)$ & $5(1.4)$ & 1.84 \\
\hline Urticaceae & $4(2.1)$ & $5(1.4)$ & 1.51 \\
\hline Equisetaceae & $1(0.5)$ & $4(1.1)$ & 1.21 \\
\hline Lemnaceae & $3(1.6)$ & $4(1.1)$ & 1.44 \\
\hline Lythraceae & $3(1.6)$ & $4(1.1)$ & 1.13 \\
\hline Onagraceae & $2(1.1)$ & $4(1.1)$ & 1.04 \\
\hline Pontederiaceae & $2(1.1)$ & $4(1.1)$ & 1.83 \\
\hline Trapaceae & $1(0.5)$ & $4(1.1)$ & 1.75 \\
\hline Acanthaceae & $3(1.6)$ & $3(0.9)$ & 1.00 \\
\hline Caryophllaceae & $3(1.6)$ & $3(0.9)$ & 1.33 \\
\hline Nymphaeaceae & $3(1.6)$ & $3(0.9)$ & 2.71 \\
\hline Plantaginaceae & $1(0.5)$ & $3(0.9)$ & 1.23 \\
\hline Acoraceae & $1(0.5)$ & $2(0.6)$ & 1.28 \\
\hline Amaranthaceae & $1(0.5)$ & $2(0.6)$ & 2.82 \\
\hline Cannaceae & $1(0.5)$ & $2(0.6)$ & 1.42 \\
\hline Haloragaceae & $1(0.5)$ & $2(0.6)$ & 1.13 \\
\hline Lentibulariaceae & $1(0.5)$ & $2(0.6)$ & 1.00 \\
\hline Menyanthaceae & $1(0.5)$ & $2(0.6)$ & 2.06 \\
\hline Solanaceae & $2(1.1)$ & $2(0.6)$ & 1.50 \\
\hline Valerianaceae & $1(0.5)$ & $2(0.6)$ & 1.00 \\
\hline Verbenaceae & $2(1.1)$ & $2(0.6)$ & 1.00 \\
\hline Violaceae & $1(0.5)$ & $2(0.6)$ & 1.00 \\
\hline Amaryllidaceae & $1(0.5)$ & $1(0.3)$ & 2.50 \\
\hline
\end{tabular}

Table 1 The taxonomic composition of wetland plants used traditionally and family use values (FUV) based on literature research (Continued)

\begin{tabular}{|c|c|c|c|}
\hline Apocynaceae & $1(0.5)$ & $1(0.3)$ & 2.67 \\
\hline Azollaceae & $1(0.5)$ & $1(0.3)$ & 2.22 \\
\hline Butomaceae & $1(0.5)$ & $1(0.3)$ & 1.50 \\
\hline Cabombaceae & $1(0.5)$ & $1(0.3)$ & 1.00 \\
\hline Campanulaceae & $1(0.5)$ & $1(0.3)$ & 1.00 \\
\hline Ceratophyllaceae & $1(0.5)$ & $1(0.3)$ & 1.71 \\
\hline Cucurbitaceae & $1(0.5)$ & $1(0.3)$ & 3.20 \\
\hline Euphorbiaceae & $1(0.5)$ & $1(0.3)$ & 1.00 \\
\hline Gentianaceae & $1(0.5)$ & $1(0.3)$ & 1.00 \\
\hline Geraniaceae & $1(0.5)$ & $1(0.3)$ & 1.00 \\
\hline Iridaceae & $1(0.5)$ & $1(0.3)$ & 1.25 \\
\hline Juncaceae & $1(0.5)$ & $1(0.3)$ & 1.71 \\
\hline Marsileaceae & $1(0.5)$ & $1(0.3)$ & 1.71 \\
\hline Menispermaceae & $1(0.5)$ & $1(0.3)$ & 1.00 \\
\hline Nelumbonaceae & $1(0.5)$ & $1(0.3)$ & 2.22 \\
\hline Papaveraceae & $1(0.5)$ & $1(0.3)$ & 1.00 \\
\hline Parkeriaceae & $1(0.5)$ & $1(0.3)$ & 1.33 \\
\hline Penthoraceae & $1(0.5)$ & $1(0.3)$ & 2.33 \\
\hline Phytolaccaceae & $1(0.5)$ & $1(0.3)$ & 2.75 \\
\hline Plumbaginaceae & $1(0.5)$ & $1(0.3)$ & 1.00 \\
\hline Salviniaceae & $1(0.5)$ & $1(0.3)$ & 1.89 \\
\hline Saururaceae & $1(0.5)$ & $1(0.3)$ & 1.33 \\
\hline Saxifragaceae & $1(0.5)$ & $1(0.3)$ & 1.67 \\
\hline Schizaeaceae & $1(0.5)$ & $1(0.3)$ & 1.50 \\
\hline
\end{tabular}

Different families made very different contribution to different use categories (Figure 1). Over half of the families contributed to the top three categories that were medicine (97\% of families), food (62.1\%) and forage (59.1\%); about one-third contributed to each of green manure, ornamental, and industrial use. However, other categories of use were more obviously concentrated within certain families. For example, fewer than $20 \%$ of families contributed to fiber use, pesticides, liquor-making, or environmental use. Nearly half of species providing pesticides were in the Polygonaceae and $54.2 \%$ of those used for environmental protection (such as preventing soil erosion and stabilizing dams) in the Poaceae. Species of the Poaceae and Cyperaceae contributed greatly to the fiber group (63.5\% of all species so used), while those in the Polygonaceae, Poaceae, and Trapaceae were well represented in liquor-making (54\% of species used). Genera showed similar patterns to those shown by families. The results as a whole showed that the top three use categories of medicine, food and forage made 
Table 2 Use categories of wetland plants and the numbers of related species

\begin{tabular}{lcc}
\hline Type of use & No. of species & Percentage of the total (\%) \\
\hline Medicine & 263 & 75.1 \\
Forage & 173 & 49.4 \\
Food & 101 & 28.9 \\
Green manure & 53 & 15.1 \\
Fiber & 52 & 14.9 \\
Industrial raw material & 48 & 13.7 \\
Ornamental & 32 & 9.1 \\
Pesticide & 26 & 7.4 \\
Environmental use & 24 & 6.9 \\
Liquor -making material & 22 & 6.3 \\
Other & 15 & 4.3 \\
\hline
\end{tabular}

use of a broader spectrum of plants taxonomically than other uses.

Some of the top families contributed greatly to some of the use categories (Table 3). The top ten families contributed about half of all species used medicinally, over $66 \%$ of those providing fodder (though lacking any contribution from Ranunculaceae) and nearly fifty percent of those used as food. Seven of the top ten families contributed $52.8 \%$ of species used as green manure. The top ten families together contributed $73 \%$ of species used for fiber, although actually only three (Poaceae, Cyperaceae, and Fabaceae) made substantial contributions. Similar patterns were apparent in the other use categories. Some top families, such as Lamiaceae, Hydrocharitaceae, Potamogetonaceae, and Brassicaceae, contributed only to certain of the major categories of use, for example Rancunculaceae (one of the top ten families) was only used for medicine, food and pesticide. Thus, families with large numbers of species used did not necessarily contribute to all categories of use.

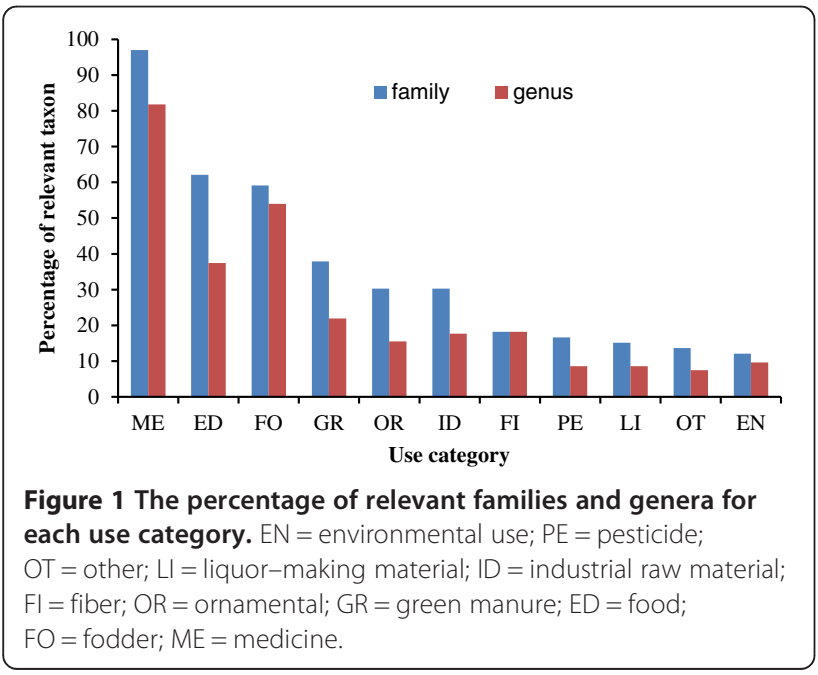

\section{Use value of wetland plants}

The Use Values (UV) of species are shown on Table 4. UV varies between 1.0 and 3.71, with ten species having $\mathrm{UV} \geq 3.00$. Phragmites australis, which was highest scoring (UV = 3.71), is one of the dominant species of wetland plant communities in China and distributed widely in many parts of the country. At least seven types of use for this species are mentioned frequently in the literature. Glycine soja was second in rank order (UV=3.5), followed by Zizania latifolia and Rorippa islandica (both $\mathrm{UV}=3.33)$, Actinostemma tenerum (UV=3.2), Rumex acetosa $(\mathrm{UV}=3.17)$ and Nymphaea tetragona $(\mathrm{UV}=3.13)$. Among species with a UV value of 3.00, Euryale ferox was recorded in ten literature sources, Saccharum spontaneum in three and Oenanthe sinensis in one; all are plants with multiple uses. There were 165 species (about $47 \%$ of the total) with the lowest possible score $(U V=1.0)$. Among these, one hundred and thirtynine species $(84.2 \%)$ had only one type of use and twenty-two species (13.3\%) had two.

Relative Frequency of Citation (RFC) varied between 0.02 (55 species, nearly $20 \%$ of the total) to 0.3 (Table 4 ). The top three species based on RFC were Polygonum hydropiper $(\mathrm{RFC}=0.3)$, Oenanthe javanica $(\mathrm{RFC}=0.25)$ and Mentha haplocalyx ( $\mathrm{RFC}=0.21)$. The next highest score was for Acorus calamus $(\mathrm{RFC}=0.20$ ) (Table 4). Many species with high RFC scores were likely to be used over extensive geographical areas, while many of those scoring just 0.02 were likely to be used only very locally.

Cultural Importance scores $(\mathrm{CI})$ ranged between 0.02 (48 species, including Rungia chinensis and A. gramineum) and 0.54 (E. ferox and Z. latifolia) (Table 4), while those for Cultural Value (CV) ranged from 0.00003 (the same 48 species as for CI) and 0.07096 (Polygonum hydropiper) (Table 4). Species with the lowest CI or CV scores had only one kind of use and were mentioned only in one literature source.

Significant correlations were found between the scores of species for RFC, CI, and CV (Figures 2, 3 and 4), once the data had been normalized appropriately. Five of the top ten species according to RFC also appeared in the top ten lists for CI and CV. These species are Polygonum hydropiper, P. orientale, Euryale ferox, Zizania latifolia, and Coix lacryma-jobi. The 48 species with the lowest CV scores were also lowest according to UV, RFC and CI.

\section{Discussion}

Diversity of wetland useful plant species

The 350 wetland species recorded as traditionally used in China according to the literature are distributed unevenly across 66 families. The top families are Poaceae, Cyperaceae, Polygonaceae, Lamiaceae, Asteraceae, Rannunculaceae, Hydrocharitaceae and Potamogetonaceae. An uneven 
Table 3 Contributions of the top 10 families (in terms of numbers of species) to different use categories

\begin{tabular}{lccccccccccc}
\hline Family & ME (\%) & FO (\%) & ED (\%) & GR (\%) & FI (\%) & ID (\%) & OR (\%) & PE (\%) & EN (\%) & LI (\%) & OT (\%) \\
\hline Poaceae & $14(5.3)$ & $42(24.3)$ & $7(6.9)$ & $2(3.8)$ & $18(34.6)$ & $2(4.2)$ & $4(12.5)$ & $0(0.0)$ & $13(54.2)$ & $4(18.2)$ & $6(40.0)$ \\
Polygonaceae & $32(12.2)$ & $11(6.4)$ & $11(10.9)$ & $1(1.9)$ & $0(0.0)$ & $14(29.2)$ & $1(3.1)$ & $12(46.2)$ & $1(4.2)$ & $5(22.7)$ & $1(6.7)$ \\
Cyperaceae & $17(6.5)$ & $19(11.0)$ & $3(3.0)$ & $1(1.9)$ & $15(28.8)$ & $1(2.1)$ & $2(6.3)$ & $0(0.0)$ & $2(8.3)$ & $2(9.1)$ & $0(0.0)$ \\
Lamiaceae & $20(7.6)$ & $2(1.2)$ & $5(5.0)$ & $0(0.0)$ & $0(0.0)$ & $4(8.3)$ & $0(0.0)$ & $1(3.8)$ & $0(0.0)$ & $0(0.0)$ & $0(0.0)$ \\
Asteraceae & $13(4.9)$ & $6(3.5)$ & $7(6.9)$ & $2(3.8)$ & $0(0.0)$ & $1(2.1)$ & $0(0.0)$ & $1(3.8)$ & $1(4.2)$ & $1(4.5)$ & $0(0.0)$ \\
Ranunculaceae & $13(4.9)$ & $0(0.0)$ & $1(1.0)$ & $0(0.0)$ & $0(0.0)$ & $0(0.0)$ & $0(0.0)$ & $5(19.2)$ & $0(0.0)$ & $0(0.0)$ & $0(0.0)$ \\
Hydrocharitaceae & $3(1.1)$ & $12(6.9)$ & $3(3.0)$ & $7(13.2)$ & $0(0.0)$ & $0(0.0)$ & $1(3.1)$ & $0(0.0)$ & $0(0.0)$ & $0(0.0)$ & $0(0.0)$ \\
Potamogetonaceae & $5(1.9)$ & $10(5.8)$ & $1(1.0)$ & $7(13.2)$ & $0(0.0)$ & $0(0.0)$ & $0(0.0)$ & $0(0.0)$ & $0(0.0)$ & $0(0.0)$ & $0(0.0)$ \\
Fabaceae & $9(3.4)$ & $8(4.6)$ & $1(1.0)$ & $8(15.1)$ & $5(9.6)$ & $2(4.2)$ & $0(0.0)$ & $0(0.0)$ & $4(16.7)$ & $0(0.0)$ & $0(0.0)$ \\
Brassicaceae & $8(3.0)$ & $5(2.9)$ & $8(7.9)$ & $0(0.0)$ & $0(0.0)$ & $5(10.4)$ & $1(3.1)$ & $0(0.0)$ & $0(0.0)$ & $0(0.0)$ & $0(0.0)$ \\
Total & $134(51.0)$ & $115(66.5)$ & $47(46.5)$ & $28(52.8)$ & $38(73.0)$ & $29(60.4)$ & $9(28.1)$ & $19(73.1)$ & $21(87.5)$ & $12(54.5)$ & $7(46.7)$ \\
\hline
\end{tabular}

Note: $M E=$ medicine; $E D=$ food; $F O=$ fodder; $G R=$ green manure; $O R=$ ornamental; $I D=$ industrial raw material; $F I=$ fiber; $P E=$ pesticide; $L I=$ liquor-making

material; $O T=$ other; $E N=$ environmental use.

distribution of useful wetland species by plant family has also been found elsewhere in the world $[11,88-90]$, for instance in Manipur (India) where Jain et al. found that Polygonaceae, Araceae, Cyperaceae and Poaceae contributed disproportionately to the list of useful species [11]. Coincidentally, many of the top families found in the Manipur study are also dominant or abundant in wetland plant communities in many parts of China [91-96]. Species scoring highly in our study and which also have wide distributions elsewhere in the world, such as Phragmites australis, Polygonum hydropiper and Zizania latifolia, are always mentioned frequently in the literature from other places. All have high UV, CI and CV values according to our study (Table 4). This suggests that families rich in wetland species are more likely to be used than others, the key factor being the local presence of species potentially available for people's attention and possible use. This result is similar to those reported for other regions [23]. Moerman et al. have argued in the case of medicinal plants that the characteristics of the local flora have a big influence on people's knowledge [97]. The more often people come into contact with particular elements of the flora, the more likely they are to find uses for them. Knowledge about the usefulness of such plants will tend to grow disproportionately, as experience is accumulated. Traditional knowledge is always related to local people's contact with the local environment [23].

\section{The characteristics of usage of wetland useful species}

Our results show that wetland plants have been used for multiple purposes in most parts of China. The three most important uses are provision of medicine, food and fodder (Table 2), all required regularly by people as they go about their daily lives $[11,13,98,99]$. Providing people with sources of green manure is a further noteworthy use made of wetland plants, with 53 species being used. Adding fertility to the soil is a basic necessity in China, which remains fundamentally an agricultural country. Providing people with sources of fiber is another regular use made of wetland plants. People in China have had a long history of using plant fiber for making cloth, rope and other articles and a rich store of knowledge about the use of wetland plants for fiber extraction and use has been accumulated by people living in and around wetlands [100].

Besides providing local people with material necessities for their everyday lives, wetland plants also provide other products used less frequently, as well as a range of services. Some plants are used as ornamentals, such as Polygonum orientale. Phragmites australis, Miscanthus sinensis, Miscanthus sacchariflorus, while others are important for the strengthening of embankments and protecting soil erosion. Twenty-two species provide raw materials for making wine. China has a cornucopia of traditional knowledge relating to liquormaking; our results confirm that a substantial part of this knowledge relates to wetland species, even though much of this knowledge is historical and not known by current generations. Wetlands can be breeding grounds for mosquitoes and other nuisance insects, reducing agricultural production or transmitting disease, so considerable traditional knowledge of wetland plants relating to pesticides may yet prove to be useful in the modern world. There are also some species having important cultural values, for example the flowers of $\mathrm{Zan}$ tedeschia aethiopica used commonly in sacrificial rites.

Compared with the uses mentioned above, the use of wetland plants for industrial purpose is comparatively recent. Industrially, wetland plants are mostly used as sources of industrial raw materials. For instance, Scirpus yagara is used as a raw material in the production of ethyl alcohol and glycerol, while Mentha haplocalyx can be a source of volatile oils. These plants can be important source of cash for local people. 
Table 4 Ethnobotanical inventory and some quantitative indexes of useful wetland plants in China

\begin{tabular}{|c|c|c|c|c|c|c|}
\hline Species & UV & RFC & $\mathrm{Cl}$ & $\mathrm{CV}$ & Use & Reference(s) \\
\hline Acorus calamus $\mathrm{L}$. & 1.55 & 0.20 & 0.30 & 0.03795 & $\mathrm{ED}, \mathrm{ME}, \mathrm{FI}, \mathrm{FO}, \mathrm{PE}, \mathrm{OR}, \mathrm{ID}$ & {$[27-29,45,46,59,63,65,68,62,67]$} \\
\hline Acorus gramineus Aiton & 1.00 & 0.11 & 0.11 & 0.00209 & $\mathrm{ED}, \mathrm{ME}$ & {$[27,44,46,59,63,78]$} \\
\hline Actinostemma tenerum Griff. & 3.20 & 0.09 & 0.29 & 0.01160 & $\mathrm{ED}, \mathrm{ME}, \mathrm{FO}, \mathrm{GR}, \mathrm{ID}$ & {$[27-29,58,64]$} \\
\hline Adenostemma lavenia (L.) Kuntze & 1.00 & 0.07 & 0.07 & 0.00046 & ED & {$[27,29,46,58]$} \\
\hline Aeginetia indica $\mathrm{L}$. & 1.00 & 0.05 & 0.05 & 0.00026 & ME & {$[28,29,46]$} \\
\hline Aeschynomene indica $\mathrm{L}$. & 2.00 & 0.05 & 0.11 & 0.00157 & $\mathrm{ME}, \mathrm{GR}, \mathrm{FI}$ & {$[29,46,58]$} \\
\hline Ageratum conyzoides L. & 2.00 & 0.05 & 0.11 & 0.00209 & $\mathrm{ME}, \mathrm{FO}, \mathrm{GR}, \mathrm{EN}$ & {$[28,29,46]$} \\
\hline Ajuga ciliata Bunge & 1.00 & 0.05 & 0.05 & 0.00026 & ME & {$[28,29,46]$} \\
\hline Ajuga multiflora Bunge & 1.00 & 0.04 & 0.04 & 0.00012 & ME & {$[28,29]$} \\
\hline Alisma canaliculatum A. Braun \& C. D. Bouché & 1.50 & 0.04 & 0.05 & 0.00035 & $\mathrm{ME}, \mathrm{OR}$ & {$[29,46]$} \\
\hline Alisma gramineum Lej. & 1.00 & 0.02 & 0.02 & 0.00003 & ME & [64] \\
\hline Alisma plantago-aquatica $\mathrm{L}$. & 1.13 & 0.14 & 0.16 & 0.00417 & $\mathrm{ME}, \mathrm{OR}$ & {$[27-29,45,46,59,64,65]$} \\
\hline Alternanthera philoxeroides (Mart.) Griseb. & 2.83 & 0.11 & 0.30 & 0.01183 & $\mathrm{ME}, \mathrm{GR}, \mathrm{FO}, \mathrm{EN}$ & {$[27-29,46,57,58]$} \\
\hline Alternanthera sessilis (L.) DC. & 2.80 & 0.09 & 0.25 & 0.00812 & $\mathrm{ED}, \mathrm{ME}, \mathrm{FO}, \mathrm{GR}$ & {$[27,29,46,58,62]$} \\
\hline Amethystea coerulea $\mathrm{L}$. & 1.00 & 0.04 & 0.04 & 0.00012 & ME & {$[28,29]$} \\
\hline Ammannia baccifera $\mathrm{L}$. & 1.00 & 0.04 & 0.04 & 0.00023 & $\mathrm{ME}, \mathrm{FO}$ & {$[46,62]$} \\
\hline Amphicarpaea trisperma Baker & 1.00 & 0.02 & 0.02 & 0.00003 & $\mathrm{FO}$ & {$[28]$} \\
\hline Anemone hupehensis (Lemoine) Lemoine & 1.67 & 0.05 & 0.09 & 0.00087 & $\mathrm{ME}, \mathrm{PE}$ & {$[28,29,46]$} \\
\hline Apium leptophyllum (Pers.) F. Muell. & 1.00 & 0.02 & 0.02 & 0.00003 & $\mathrm{FO}$ & [28] \\
\hline Apocynum venetum $\mathrm{L}$. & 2.67 & 0.05 & 0.14 & 0.00278 & $\mathrm{ED}, \mathrm{ME}, \mathrm{FI}, \mathrm{ID}$ & {$[28,29,46]$} \\
\hline Arisaema amurense Maxim. & 1.00 & 0.07 & 0.07 & 0.00046 & ME & {$[27,28,46,59]$} \\
\hline Arisaema du-bois-reymondiae Engl. & 1.00 & 0.02 & 0.02 & 0.00003 & ME & [29] \\
\hline Arisaema heterophyllum Blume & 1.00 & 0.02 & 0.02 & 0.00003 & ME & {$[69]$} \\
\hline Artemisia capillaris Thunb. & 2.20 & 0.09 & 0.20 & 0.00957 & $\mathrm{ED}, \mathrm{ME}, \mathrm{FO}, \mathrm{LI}, \mathrm{PE}, \mathrm{ID}$ & {$[27-29,58,67]$} \\
\hline Artemisia selengensis Turcz. ex Besser & 1.60 & 0.09 & 0.14 & 0.00348 & $\mathrm{ED}, \mathrm{ME}, \mathrm{FO}$ & {$[27,29,46,65,70]$} \\
\hline Arthraxon hispidus (Thunb.) Makino & 1.50 & 0.07 & 0.11 & 0.00209 & $\mathrm{ME}, \mathrm{FI}, \mathrm{FO}$ & {$[28,46,57,67]$} \\
\hline Arundinella anomala Steud. & 2.00 & 0.04 & 0.07 & 0.00046 & $\mathrm{Fl}, \mathrm{FO}$ & {$[28,29]$} \\
\hline Arundo donax L. & 2.63 & 0.14 & 0.38 & 0.02922 & $\mathrm{ME}, \mathrm{Fl}, \mathrm{FO}, \mathrm{OR}, \mathrm{EN}, \mathrm{OT}$ & {$[27-29,36,37,45,46,59]$} \\
\hline Arundo donax var. versicolor (Mill.) Stokes & 1.00 & 0.04 & 0.04 & 0.00012 & OR & {$[27,38]$} \\
\hline Astilbe chinensis Franch. \& Sav. & 1.67 & 0.05 & 0.09 & 0.00130 & $M E, O R, I D$ & {$[28,29,46]$} \\
\hline Astragalus adsurgens Pall. & 1.67 & 0.05 & 0.09 & 0.00130 & $\mathrm{ME}, \mathrm{FO}, \mathrm{EN}$ & {$[28,29,46]$} \\
\hline Atropanthe sinensis Pascher & 1.67 & 0.05 & 0.09 & 0.00087 & $M E, I D$ & {$[28,29,46]$} \\
\hline Azolla imbricata (Roxb.) Nakai & 2.22 & 0.16 & 0.36 & 0.02087 & $\mathrm{ME}, \mathrm{FO}, \mathrm{GR}, \mathrm{PE}$ & {$[27-29,31,32,46,58,59,62]$} \\
\hline Bacopa monnieri (L.) Wettst. & 1.00 & 0.04 & 0.04 & 0.00012 & ME & {$[28,29]$} \\
\hline Beckmannia syzigachne (Steud.) Fernald & 1.67 & 0.05 & 0.09 & 0.00130 & $\mathrm{ED}, \mathrm{ME}, \mathrm{FO}$ & {$[28,57,68]$} \\
\hline Berteroa incana DC. & 1.00 & 0.02 & 0.02 & 0.00003 & ID & {$[28]$} \\
\hline Bidens parviflora Willd. & 1.33 & 0.05 & 0.07 & 0.00070 & $\mathrm{ED}, \mathrm{ME}$ & {$[28,29,46]$} \\
\hline Bidens tripartita $\mathrm{L}$. & 1.00 & 0.05 & 0.05 & 0.00026 & ME & {$[28,29,46]$} \\
\hline Blyxa aubertii Rich. & 1.00 & 0.02 & 0.02 & 0.00003 & $\mathrm{FO}$ & {$[28]$} \\
\hline Blyxa echinosperma (C. B. Clarke) Hook. f. & 1.00 & 0.04 & 0.04 & 0.00012 & $\mathrm{FO}$ & {$[27,28]$} \\
\hline Blyxa japonica Maxim. ex Asch. \& Gürke & 1.00 & 0.02 & 0.02 & 0.00003 & FO & [28] \\
\hline Blyxa leiosperma Koidz. & 1.00 & 0.02 & 0.02 & 0.00003 & $\mathrm{FO}$ & {$[27]$} \\
\hline Boehmeria gracilis C. H. Wright & 1.67 & 0.05 & 0.09 & 0.00130 & $M E, F I, I D$ & {$[28,29,46]$} \\
\hline Brasenia schreberi J. F. Gmel. & 1.00 & 0.05 & 0.05 & 0.00052 & $\mathrm{ED}, \mathrm{ME}$ & {$[27,29,46]$} \\
\hline
\end{tabular}




\section{Table 4 Ethnobotanical inventory and some quantitative indexes of useful wetland plants in China (Continued)}

\begin{tabular}{|c|c|c|c|c|c|c|}
\hline Bromus catharticus Vahl & 1.00 & 0.04 & 0.04 & 0.00012 & $\mathrm{FO}$ & {$[27,29]$} \\
\hline Bromus inermis Leyss. & 2.50 & 0.04 & 0.09 & 0.00087 & $\mathrm{ED}, \mathrm{FO}, \mathrm{EN}$ & {$[27,28]$} \\
\hline Bromus japonicus Thunb. & 1.83 & 0.11 & 0.20 & 0.00957 & $\mathrm{ED}, \mathrm{ME}, \mathrm{FO}, \mathrm{Fl}, \mathrm{LI}$ & {$[27,28,45,46,57,62]$} \\
\hline Bromus remotiflorus (Steud.) Ohwi & 1.33 & 0.05 & 0.07 & 0.00070 & $\mathrm{Fl}, \mathrm{FO}$ & {$[45,57,62]$} \\
\hline Butomus umbellatus $\mathrm{L}$. & 1.50 & 0.04 & 0.05 & 0.00035 & $\mathrm{Fl}, \mathrm{OR}$ & {$[28,64]$} \\
\hline Calamagrostis epigeios (L.) Roth & 2.60 & 0.09 & 0.23 & 0.00754 & $\mathrm{Fl}, \mathrm{FO}, \mathrm{OT}, \mathrm{EN}$ & {$[27-29,57,67]$} \\
\hline Calamagrostis pseudophragmites (Hall. f.) Koel. & 2.00 & 0.07 & 0.14 & 0.00186 & FO,EN & {$[27-29,57]$} \\
\hline Caldesia reniformis Makino & 1.00 & 0.02 & 0.02 & 0.00003 & OR & [29] \\
\hline Caltha palustris $\mathrm{L}$. & 1.33 & 0.05 & 0.07 & 0.00104 & $\mathrm{ED}, \mathrm{ME}, \mathrm{PE}$ & {$[29,68,82]$} \\
\hline Canna generalis L. H. Bailey & 1.50 & 0.04 & 0.05 & 0.00035 & $\mathrm{Fl}, \mathrm{OR}$ & {$[27,29]$} \\
\hline Canna indica $\mathrm{L}$. & 1.33 & 0.05 & 0.07 & 0.00139 & $\mathrm{ED}, \mathrm{ME}, \mathrm{Fl}, \mathrm{FO}$ & {$[27,46,62]$} \\
\hline Capillipedium parviflorum (R. Br.) Stapf & 1.00 & 0.02 & 0.02 & 0.00003 & FO & [28] \\
\hline Cardamine flexuosa With. & 1.00 & 0.05 & 0.05 & 0.00026 & ME & {$[28,29,58]$} \\
\hline Cardamine impatiens $\mathrm{L}$. & 1.75 & 0.07 & 0.13 & 0.00244 & $\mathrm{ED}, \mathrm{ME}, \mathrm{FO}$ & {$[27-29,63]$} \\
\hline Cardamine leucantha (Tausch) O. E. Schulz & 1.25 & 0.07 & 0.09 & 0.00116 & $\mathrm{ED}, \mathrm{ME}$ & {$[28,29,46,70]$} \\
\hline Cardamine lyrata Bunge & 1.75 & 0.07 & 0.13 & 0.00162 & $\mathrm{ED}, \mathrm{ME}$ & {$[27-29,46]$} \\
\hline Cardamine macrophylla Willd. & 1.67 & 0.05 & 0.09 & 0.00130 & $E D, M E, F O$ & {$[29,81,82]$} \\
\hline Carex baccans Nees & 1.00 & 0.04 & 0.04 & 0.00023 & $\mathrm{ED}, \mathrm{ME}$ & {$[28,46]$} \\
\hline Carex dispalata Boott & 1.50 & 0.04 & 0.05 & 0.00035 & $\mathrm{Fl}, \mathrm{FO}$ & {$[28,67]$} \\
\hline Carex leiorhyncha C. A. Mey. & 1.00 & 0.04 & 0.04 & 0.00012 & FO & {$[28,67]$} \\
\hline Carex scabrifolia Steud. & 1.00 & 0.04 & 0.04 & 0.00012 & $\mathrm{FI}$ & {$[28,29]$} \\
\hline Carex tangiana Ohwi & 1.50 & 0.04 & 0.05 & 0.00035 & $\mathrm{FO}, \mathrm{OR}$ & {$[28,67]$} \\
\hline Catabrosa aquatica P. Beauv. & 1.00 & 0.02 & 0.02 & 0.00003 & $\mathrm{FO}$ & [28] \\
\hline Centaurium meyeri Druce & 1.00 & 0.04 & 0.04 & 0.00012 & ME & {$[28,46]$} \\
\hline Centipeda minima (L.) A. Braun \& Asch. & 1.00 & 0.07 & 0.07 & 0.00046 & ME & {$[28,29,46,78]$} \\
\hline Ceratophyllum demersum $\mathrm{L}$. & 1.71 & 0.13 & 0.21 & 0.00487 & $\mathrm{ME}, \mathrm{FO}$ & {$[27-29,46,58,63,64]$} \\
\hline Ceratopteris thalictroides (L.) Brongn. & 1.33 & 0.11 & 0.14 & 0.00278 & $\mathrm{ED}, \mathrm{ME}$ & {$[27-29,46,58,63]$} \\
\hline Chenopodium ambrosioides $\mathrm{L}$. & 2.20 & 0.09 & 0.20 & 0.00638 & $M E, E D, P E, I D$ & {$[27-29,46,58]$} \\
\hline Chenopodium serotinum $\mathrm{L}$. & 1.00 & 0.04 & 0.04 & 0.00023 & $\mathrm{ME}, \mathrm{FO}$ & {$[46,62]$} \\
\hline Cicuta virosa $L$. & 1.00 & 0.04 & 0.04 & 0.00012 & ME & {$[28,46]$} \\
\hline Clematis cadmia Buch.-Ham. ex Hook.f. \& Thomson & 1.00 & 0.04 & 0.04 & 0.00012 & ME & {$[28,46]$} \\
\hline Clematis finetiana $\mathrm{H}$. Lév. \& Vaniot & 1.00 & 0.05 & 0.05 & 0.00026 & ME & {$[28,29,46]$} \\
\hline Clematis orientalis $\mathrm{L}$. & 1.00 & 0.02 & 0.02 & 0.00003 & ME & [28] \\
\hline Clinopodium chinense Kuntze & 1.00 & 0.04 & 0.04 & 0.00012 & ME & {$[28,46]$} \\
\hline Clinopodium gracile (Bentham) Matsumura & 1.00 & 0.05 & 0.05 & 0.00026 & ME & {$[28,29,46]$} \\
\hline Cnidium monnieri (L.) Cuss. & 1.33 & 0.05 & 0.07 & 0.00070 & $\mathrm{ME}, \mathrm{FO}$ & {$[28,29,46]$} \\
\hline Coix lacryma-jobi L. & 2.30 & 0.18 & 0.41 & 0.04000 & $\mathrm{ED}, \mathrm{ME}, \mathrm{FI}, \mathrm{FO}, \mathrm{LI}, \mathrm{OT}$ & {$[27,41,42,45,46,59,62,63,65,71]$} \\
\hline Colocasia esculenta (L.) Schott & 1.83 & 0.11 & 0.20 & 0.00574 & $\mathrm{ED}, \mathrm{ME}, \mathrm{FO}$ & {$[27,29,46,65,59,62]$} \\
\hline Commelina benghalensis $\mathrm{L}$. & 1.00 & 0.07 & 0.07 & 0.00093 & $\mathrm{ME}, \mathrm{OR}$ & {$[27-29,46]$} \\
\hline Commelina communis L. & 1.20 & 0.09 & 0.11 & 0.00261 & $\mathrm{ED}, \mathrm{ME}, \mathrm{FO}$ & {$[27-29,46,70]$} \\
\hline Corydalis racemosa Pers. & 1.00 & 0.07 & 0.07 & 0.00046 & ME & {$[28,29,46,58]$} \\
\hline Crotalaria assamica Benth. & 2.00 & 0.04 & 0.07 & 0.00093 & $\mathrm{ME}, \mathrm{Fl}, \mathrm{FO}, \mathrm{GR}$ & {$[28,29]$} \\
\hline Crypsis aculeata Aiton & 1.50 & 0.04 & 0.05 & 0.00035 & FO,EN & {$[28,29]$} \\
\hline Cyperus compressus L. & 1.00 & 0.04 & 0.04 & 0.00012 & $\mathrm{FO}$ & {$[62,67]$} \\
\hline Cyperus difformis L. & 1.00 & 0.11 & 0.11 & 0.00209 & $\mathrm{ME}, \mathrm{Fl}$ & {$[28,46,62-64,67]$} \\
\hline
\end{tabular}




\section{Table 4 Ethnobotanical inventory and some quantitative indexes of useful wetland plants in China (Continued)}

\begin{tabular}{|c|c|c|c|c|c|c|}
\hline Cyperus exaltatus Retz. & 1.00 & 0.07 & 0.07 & 0.00093 & $\mathrm{Fl}, \mathrm{FO}$ & {$[28,29,62,67]$} \\
\hline Cyperus glomeratus L. & 1.60 & 0.09 & 0.14 & 0.00464 & $\mathrm{ME}, \mathrm{FO}, \mathrm{FI}, \mathrm{GR}$ & {$[28,46,62,64,67]$} \\
\hline Cyperus imbricatus Retz. & 1.00 & 0.04 & 0.04 & 0.00012 & $\mathrm{Fl}$ & {$[28,29]$} \\
\hline Cyperus iria L. & 1.00 & 0.05 & 0.05 & 0.00052 & $\mathrm{ME}, \mathrm{FO}$ & {$[46,62,67]$} \\
\hline Cyperus michelianus (L.) Link & 1.00 & 0.02 & 0.02 & 0.00003 & ME & {$[46]$} \\
\hline Cyperus microiria Steud. & 1.00 & 0.02 & 0.02 & 0.00003 & $\mathrm{FO}$ & {$[62]$} \\
\hline Cyperus pilosus Vahl & 1.00 & 0.07 & 0.07 & 0.00139 & $\mathrm{ME}, \mathrm{Fl}, \mathrm{FO}$ & {$[27,45,62,63]$} \\
\hline Cyperus pygmaeus Rottb. & 1.00 & 0.02 & 0.02 & 0.00003 & $\mathrm{FO}$ & {$[62]$} \\
\hline Dichrocephala auriculata Druce & 1.00 & 0.05 & 0.05 & 0.00026 & ME & {$[28,29,46]$} \\
\hline Dichrocephala benthamii C. B. Clarke & 1.00 & 0.05 & 0.05 & 0.00026 & ME & {$[28,29,46]$} \\
\hline Dicliptera chinensis (L.) Juss. & 1.00 & 0.05 & 0.05 & 0.00026 & ME & {$[28,29,46]$} \\
\hline Duchesnea indica (Andrews) Focke & 1.20 & 0.09 & 0.11 & 0.00261 & $\mathrm{ED}, \mathrm{ME}, \mathrm{PE}$ & {$[28,29,46,69,78]$} \\
\hline Echinochloa caudata Roshev. & 1.00 & 0.02 & 0.02 & 0.00003 & $\mathrm{FO}$ & {$[28]$} \\
\hline Echinochloa crus-galli (L.) P. Beauv. & 2.40 & 0.09 & 0.21 & 0.01044 & $\mathrm{ED}, \mathrm{ME}, \mathrm{Fl}, \mathrm{FO}, \mathrm{GR}, \mathrm{LI}$ & {$[28,45,46,57,62]$} \\
\hline Echinochloa crus-galli var. mitis (Pursh) Peterm. & 1.00 & 0.02 & 0.02 & 0.00003 & $\mathrm{FO}$ & {$[62]$} \\
\hline Echinochloa crus-galli var. zelayensis (Kunth) Hitchc. & 1.00 & 0.02 & 0.02 & 0.00003 & $\mathrm{FO}$ & {$[62]$} \\
\hline Echinochloa crus-pavonis (Kunth) Schult. & 1.00 & 0.02 & 0.02 & 0.00003 & $\mathrm{FO}$ & {$[62]$} \\
\hline Eichhornia crassipes (Mart.) Solms & 2.67 & 0.11 & 0.29 & 0.01670 & $\mathrm{ED}, \mathrm{ME}, \mathrm{FO}, \mathrm{OR}, \mathrm{GR}, \mathrm{EN}$ & {$[27-29,46,57,62]$} \\
\hline Eleocharis dulcis Trin. ex Henschel. & 2.00 & 0.11 & 0.21 & 0.00626 & $\mathrm{ED}, \mathrm{ME}, \mathrm{FO}$ & {$[27,29,43,62,65,73]$} \\
\hline Eleocharis plantagineiformis Tang \& F. T. Wang & 1.00 & 0.02 & 0.02 & 0.00003 & $\mathrm{FO}$ & [28] \\
\hline Eleocharis valleculosa Ohwi & 1.33 & 0.05 & 0.07 & 0.00070 & $\mathrm{Fl}, \mathrm{FO}$ & {$[27,57,62]$} \\
\hline Eleocharis yokoscensis (Franch. \& Savat.) Tang \& F. T. Wang & 1.00 & 0.02 & 0.02 & 0.00003 & ME & [46] \\
\hline Elsholtzia kachinensis Prain & 1.40 & 0.09 & 0.13 & 0.00304 & $\mathrm{ED}, \mathrm{ME}, \mathrm{FO}$ & {$[28,29,46,63,72]$} \\
\hline Equisetum debile Roxb. ex Vaucher & 1.50 & 0.07 & 0.11 & 0.00139 & ME,OT & {$[27,28,46,58]$} \\
\hline Equisetum hyemale $\mathrm{L}$. & 1.33 & 0.05 & 0.07 & 0.00070 & ME,OT & {$[28,46,67]$} \\
\hline Equisetum pratense Ehrh. & 1.00 & 0.04 & 0.04 & 0.00012 & ME & {$[28,67]$} \\
\hline Equisetum ramosissimum Desf. & 1.00 & 0.05 & 0.05 & 0.00026 & ME & {$[27,28,65]$} \\
\hline Eriocaulon australe R. Br. & 1.00 & 0.04 & 0.04 & 0.00012 & ME & {$[28,46]$} \\
\hline Eriocaulon buergerianum Körn. & 1.00 & 0.11 & 0.11 & 0.00104 & ME & {$[27-29,45,46,59]$} \\
\hline Eriocaulon cinereum R. Br. & 1.00 & 0.04 & 0.04 & 0.00012 & ME & {$[28,63]$} \\
\hline Eriocaulon decemflorum Maxim. & 1.00 & 0.02 & 0.02 & 0.00003 & ME & {$[27]$} \\
\hline Eriocaulon robustius Makino & 1.00 & 0.02 & 0.02 & 0.00003 & ME & {$[28]$} \\
\hline Euphorbia thymifolia L. & 1.00 & 0.07 & 0.07 & 0.00046 & ME & {$[28,29,46,68]$} \\
\hline Euryale ferox Salisb. & 3.00 & 0.18 & 0.54 & 0.06088 & $\mathrm{ED}, \mathrm{ME}, \mathrm{FO}, \mathrm{GR}, \mathrm{LI}, \mathrm{OR}, \mathrm{ID}$ & {$[27,29,45,46,57,58,62-65]$} \\
\hline Fimbristylis miliacea (L.) Vahl & 1.67 & 0.05 & 0.09 & 0.00130 & $\mathrm{ME}, \mathrm{Fl}, \mathrm{FO}$ & {$[27,28,46]$} \\
\hline Geranium sibiricum L. & 1.00 & 0.04 & 0.04 & 0.00012 & ME & {$[28,46]$} \\
\hline Geum aleppicum Jacq. & 1.75 & 0.07 & 0.13 & 0.00244 & $E D, M E, I D$ & {$[28,29,46,67]$} \\
\hline Glaux maritima L. & 1.00 & 0.04 & 0.04 & 0.00012 & $\mathrm{ED}$ & {$[28,70]$} \\
\hline Glechoma longituba (Nakai) Kuprian. & 1.00 & 0.07 & 0.07 & 0.00046 & ME & {$[28,29,46,63]$} \\
\hline Glycine soja Siebold \& Zucc. & 3.50 & 0.07 & 0.25 & 0.00974 & $\mathrm{ED}, \mathrm{ME}, \mathrm{FI}, \mathrm{FO}, \mathrm{GR}, \mathrm{EN}$ & {$[28,29,57,67]$} \\
\hline Glycyrrhiza pallidiflora Maxim. & 1.00 & 0.05 & 0.05 & 0.00078 & $\mathrm{ME}, \mathrm{Fl}, \mathrm{GR}$ & {$[28,29,46]$} \\
\hline Halerpestes cymbalaria Greene & 1.00 & 0.02 & 0.02 & 0.00003 & ME & [28] \\
\hline Halerpestes ruthenica (Jacq.) Ovcz. & 1.00 & 0.02 & 0.02 & 0.00003 & PE & {$[28]$} \\
\hline Hemarthria altissima (Poir.) Stapf \& C. E. Hubb. & 1.50 & 0.04 & 0.05 & 0.00035 & $\mathrm{FO}, \mathrm{Fl}$ & {$[27,28]$} \\
\hline Hemarthria compressa (L. f.) R. Br. & 1.00 & 0.02 & 0.02 & 0.00003 & $\mathrm{FO}$ & {$[28]$} \\
\hline
\end{tabular}




\section{Table 4 Ethnobotanical inventory and some quantitative indexes of useful wetland plants in China (Continued)}

\begin{tabular}{|c|c|c|c|c|c|c|}
\hline Hydrilla verticillata (L. f.) Royle & 2.00 & 0.05 & 0.11 & 0.00104 & $\mathrm{FO}, \mathrm{GR}$ & {$[27,28,64]$} \\
\hline Hydrocharis dubia (Blume) Backer & 1.67 & 0.05 & 0.09 & 0.00130 & $\mathrm{ED}, \mathrm{FO}, \mathrm{GR}$ & {$[27-29]$} \\
\hline Hygrophila salicifolia (Vahl) Nees & 1.00 & 0.04 & 0.04 & 0.00012 & ME & {$[28,46]$} \\
\hline Inula japonica Thunb. & 1.00 & 0.05 & 0.05 & 0.00026 & ME & {$[28,29,46]$} \\
\hline Iris tectorum Maxim. & 1.25 & 0.07 & 0.09 & 0.00116 & $\mathrm{ME}, \mathrm{OR}$ & {$[27,29,46,59]$} \\
\hline Ixeris japonica Nakai & 1.00 & 0.04 & 0.04 & 0.00012 & ME & {$[28,46]$} \\
\hline Ixeris polycephala Cass. & 1.00 & 0.02 & 0.02 & 0.00003 & $\mathrm{ME}$ & [29] \\
\hline Juncus effusus L. & 1.71 & 0.13 & 0.21 & 0.00731 & $\mathrm{ME}, \mathrm{Fl}, \mathrm{OT}$ & {$[27-29,46,64,65,67]$} \\
\hline Kyllinga brevifolia Rottb. & 1.00 & 0.04 & 0.04 & 0.00012 & ME & {$[28,46]$} \\
\hline Kyllinga colorata (L.) Druce & 1.00 & 0.02 & 0.02 & 0.00003 & ME & [28] \\
\hline Lactuca tatarica C. A. Mey. & 2.00 & 0.02 & 0.04 & 0.00012 & $\mathrm{ED}, \mathrm{FO}$ & {$[28]$} \\
\hline Lagedium sibiricum (L.) Soják & 1.00 & 0.02 & 0.02 & 0.00003 & $\mathrm{ED}$ & [70] \\
\hline Lamium amplexicaule L. & 1.00 & 0.05 & 0.05 & 0.00026 & ME & {$[28,29,79]$} \\
\hline Lamium barbatum Siebold \& Zucc. & 1.00 & 0.05 & 0.05 & 0.00026 & ME & {$[28,29,46]$} \\
\hline Lapsana apogonoides Maxim. & 1.00 & 0.05 & 0.05 & 0.00026 & $\mathrm{FO}$ & {$[28,29,57]$} \\
\hline Leersia hexandra Sw. & 1.50 & 0.04 & 0.05 & 0.00052 & ME,FO,ID & {$[28,46]$} \\
\hline Leersia japonica Makino & 1.00 & 0.02 & 0.02 & 0.00003 & ME & [46] \\
\hline Leersia oryzoides (L.) Sw. & 1.00 & 0.02 & 0.02 & 0.00003 & $\mathrm{FO}$ & [28] \\
\hline Lemna minor $\mathrm{L}$. & 1.75 & 0.07 & 0.13 & 0.00244 & $\mathrm{ME}, \mathrm{FO}, \mathrm{GR}$ & {$[28,48,59,62]$} \\
\hline Lemna trisulca L. & 1.00 & 0.02 & 0.02 & 0.00003 & $\mathrm{FO}$ & [28] \\
\hline Leptochloa chinensis (L.) Nees & 1.00 & 0.05 & 0.05 & 0.00026 & $\mathrm{FO}$ & {$[27,29,62]$} \\
\hline Limonium sinense Kuntze & 1.00 & 0.05 & 0.05 & 0.00026 & ME & {$[28,29,46]$} \\
\hline Lobelia chinensis Lour. & 1.00 & 0.07 & 0.07 & 0.00046 & ME & {$[28,29,46,58]$} \\
\hline Lotus tenuis Waldst. \& Kit. ex Willd. & 1.00 & 0.04 & 0.04 & 0.00012 & ME & {$[28,46]$} \\
\hline Ludwigia adscendens (L.) H. Hara & 1.17 & 0.11 & 0.13 & 0.00244 & $\mathrm{ME}, \mathrm{FO}$ & {$[27-29,46,58,62]$} \\
\hline Ludwigia hyssopifolia (G. Don) Exell & 1.00 & 0.05 & 0.05 & 0.00026 & ME & {$[28,29,46]$} \\
\hline Ludwigia prostrata Roxb. & 1.00 & 0.05 & 0.05 & 0.00052 & $\mathrm{ME}, \mathrm{FO}$ & {$[27,46,62]$} \\
\hline Lycopus lucidus Turcz. & 1.20 & 0.09 & 0.11 & 0.00174 & $\mathrm{ED}, \mathrm{ME}$ & {$[28,29,46,69,70]$} \\
\hline Lycoris radiata (L'Hér.) Herb. & 2.50 & 0.07 & 0.18 & 0.00812 & ED,ME,PE,LI,FI,OT,ID & {$[27-29,46]$} \\
\hline Lygodium japonicum (Thunb.) Sw. & 1.50 & 0.07 & 0.11 & 0.00209 & ME,PE,ED & {$[28,29,46,77]$} \\
\hline Lysimachia christinae Hance & 1.00 & 0.09 & 0.09 & 0.00072 & ME & {$[27-29,46,58]$} \\
\hline Lysimachia congestiflora Hemsl. & 1.00 & 0.07 & 0.07 & 0.00046 & ME & {$[27-29,46]$} \\
\hline Lysimachia fortunei Maxim. & 1.20 & 0.09 & 0.11 & 0.00174 & $\mathrm{ME}, \mathrm{FO}$ & {$[27-29,46,58]$} \\
\hline Lysimachia heterogenea Klatt & 2.00 & 0.02 & 0.04 & 0.00012 & $M E, G R$ & [28] \\
\hline Lythrum salicaria L. & 1.50 & 0.14 & 0.21 & 0.01113 & $\mathrm{ME}, \mathrm{OR}, \mathrm{FO}, \mathrm{ID}$ & {$[27-29,46,58,62,64,65]$} \\
\hline Marsilea quadrifolia L. & 1.71 & 0.13 & 0.21 & 0.00974 & $\mathrm{ED}, \mathrm{ME}, \mathrm{FO}, \mathrm{GR}$ & {$[27-29,46,56,58,59]$} \\
\hline Mazus japonicus (Thunb.) Kuntze & 1.00 & 0.04 & 0.04 & 0.00012 & ME & {$[27,46]$} \\
\hline Melilotus indicus (L.) All. & 2.33 & 0.05 & 0.13 & 0.00244 & $\mathrm{ME}, \mathrm{FO}, \mathrm{GR}, \mathrm{EN}$ & {$[28,46,57]$} \\
\hline Mentha haplocalyx Briq. & 1.67 & 0.21 & 0.36 & 0.02087 & $\mathrm{ED}, \mathrm{ME}, \mathrm{ID}$ & {$[27-29,45,46,58,59,65,67,70,78,77]$} \\
\hline Microstegium ciliatum A. Camus & 1.50 & 0.04 & 0.05 & 0.00035 & $\mathrm{Fl}, \mathrm{FO}$ & {$[28,29]$} \\
\hline Mimulus tenellus Bunge & 1.00 & 0.05 & 0.05 & 0.00052 & $\mathrm{ED}, \mathrm{ME}$ & {$[28,29,46]$} \\
\hline Miscanthus floridulus Warb. ex K. Schum. \& Lauterb. & 1.83 & 0.11 & 0.20 & 0.00765 & $\mathrm{ME}, \mathrm{FO}, \mathrm{Fl}, \mathrm{EN}$ & {$[27-29,46,57,62]$} \\
\hline Miscanthus sacchariflorus (Maxim.) Hack. & 2.00 & 0.09 & 0.18 & 0.00435 & $\mathrm{FI}, \mathrm{FO}, \mathrm{EN}$ & {$[27,28,45,57,62]$} \\
\hline Miscanthus sinensis Andersson & 2.00 & 0.11 & 0.21 & 0.00835 & $\mathrm{ME}, \mathrm{FI}, \mathrm{FO}, \mathrm{EN}$ & {$[27-29,45,46,62]$} \\
\hline Monochoria hastata (L.) Solms & 1.00 & 0.04 & 0.04 & 0.00012 & ED & {$[28,56]$} \\
\hline
\end{tabular}




\section{Table 4 Ethnobotanical inventory and some quantitative indexes of useful wetland plants in China (Continued)}

Monochoria korsakowii Regel \& Maack

Monochoria vaginalis (Burm. f.) C. Presl ex Kunth

Mosla dianthera (Buch.-Ham. ex Roxb.) Maxim.

Murdannia keisak (Hassk.) Hand.-Mazz.

Murdannia nudiflora (L.) Brenan

Murdannia triquetra G. Brückn.

Myosoton aquaticum Moench

Myriophyllum spicatum L.

Myriophyllum verticillatum $\mathrm{L}$.

Najas foveolata A. Braun ex Magnus

Najas graminea Delile

Najas marina L.

Najas minor All.

Nanocnide japonica Blume

Nanocnide lobata Wedd.

Nasturtium officinale R. Br.

Nelumbo nucifera Gaertn.

Nepeta cataria L.

Nuphar pumila (Timm) DC.

Nymphaea tetragona Georgi

Nymphoides indica (L.) Kuntze

Nymphoides peltata (S. G. Gmel.) Kuntze

Oenanthe benghalensis Benth. \& Hook.f.

Oenanthe javanica DC.

Oenanthe sinensis Dunn

Oenothera rosea Aiton

Origanum vulgare L.

Ottelia acuminata (Gagnep.) Dandy

Ottelia alismoides (L.) Pers.

Panicum paludosum Roxb.

Paspalum dilatatum Poir.

Paspalum distichum $\mathrm{L}$.

Paspalum paspaloides Scribn.

Paspalum thunbergii Kunth ex Steud.

Penthorum chinense Pursh

Phalaris arundinacea $\mathrm{L}$.

Phragmites australis Trin. ex Steud.

Phragmites karka (Retz.) Trin. ex Steud.

Phyla nodiflora (L.) Greene

Phytolacca acinosa Roxb.

Pilea notata C. H. Wright

Pistia stratiotes L.

Plantago asiatica L.

Plantago lanceolata L.

Plantago major L.

\begin{tabular}{|c|c|c|c|c|c|}
\hline 2.00 & 0.14 & 0.29 & 0.01855 & $\mathrm{ED}, \mathrm{ME}, \mathrm{FO}, \mathrm{OR}, \mathrm{GR}$ & {$[27-29,46,56,57,62,64]$} \\
\hline 1.67 & 0.11 & 0.18 & 0.00696 & $\mathrm{ED}, \mathrm{ME}, \mathrm{FO}, \mathrm{GR}$ & {$[27,46,56,57,62,70]$} \\
\hline 1.33 & 0.05 & 0.07 & 0.00070 & ME,PE & {$[28,29,46]$} \\
\hline 1.00 & 0.02 & 0.02 & 0.00003 & $\mathrm{FO}$ & [28] \\
\hline 1.00 & 0.04 & 0.04 & 0.00012 & ME & {$[28,29]$} \\
\hline 2.00 & 0.07 & 0.14 & 0.00278 & $\mathrm{ME}, \mathrm{ED}, \mathrm{FO}$ & {$[27,29,46,49]$} \\
\hline 2.00 & 0.05 & 0.11 & 0.00157 & $\mathrm{ME}, \mathrm{ED}, \mathrm{FO}$ & {$[28,29,46]$} \\
\hline 1.25 & 0.07 & 0.09 & 0.00116 & $\mathrm{ME}, \mathrm{FO}$ & {$[27-29,64]$} \\
\hline 1.00 & 0.05 & 0.05 & 0.00026 & $\mathrm{FO}$ & {$[27,28,64]$} \\
\hline 2.00 & 0.02 & 0.04 & 0.00012 & $\mathrm{FO}, \mathrm{GR}$ & [28] \\
\hline 2.00 & 0.04 & 0.07 & 0.00046 & $\mathrm{FO}, \mathrm{GR}$ & {$[28,29]$} \\
\hline 1.50 & 0.07 & 0.11 & 0.00139 & $\mathrm{FO}, \mathrm{GR}$ & {$[27,28,62,64]$} \\
\hline 1.50 & 0.07 & 0.11 & 0.00139 & $F O, G R$ & {$[27,28,62,64]$} \\
\hline 1.00 & 0.05 & 0.05 & 0.00026 & ME & {$[27,28,46]$} \\
\hline 1.33 & 0.05 & 0.07 & 0.00070 & $\mathrm{ME}, \mathrm{GR}$ & {$[28,29,46]$} \\
\hline 2.25 & 0.07 & 0.16 & 0.00417 & $\mathrm{ED}, \mathrm{ME}, \mathrm{OR}, \mathrm{ID}$ & {$[28,46,58,65]$} \\
\hline 2.22 & 0.16 & 0.36 & 0.02087 & $\mathrm{ED}, \mathrm{ME}, \mathrm{OR}, \mathrm{FO}$ & {$[27,29,45,58,46,59,62,65,66]$} \\
\hline 1.50 & 0.07 & 0.11 & 0.00139 & $M E, I D$ & {$[28,29,46,66]$} \\
\hline 2.00 & 0.13 & 0.25 & 0.01136 & $\mathrm{ME}, \mathrm{ED}, \mathrm{OR}, \mathrm{FO}$ & {$[27,29,46,54,58,59,62]$} \\
\hline 3.13 & 0.14 & 0.45 & 0.02899 & $\mathrm{ED}, \mathrm{LI}, \mathrm{ME}, \mathrm{OR}, \mathrm{GR}$ & {$[27,29,45,46,58,62,64,65]$} \\
\hline 2.00 & 0.02 & 0.04 & 0.00012 & $\mathrm{FO}, \mathrm{GR}$ & {$[27]$} \\
\hline 2.13 & 0.14 & 0.30 & 0.01971 & $\mathrm{ME}, \mathrm{FO}, \mathrm{GR}, \mathrm{OR}, \mathrm{ED}$ & {$[27-29,46,58,59,62,70]$} \\
\hline 1.00 & 0.04 & 0.04 & 0.00012 & ME & {$[28,46]$} \\
\hline 1.36 & 0.25 & 0.34 & 0.02313 & $\mathrm{ED}, \mathrm{ME}, \mathrm{FO}$ & {$[27-29,45,46,56-58,64-66,72,70,76]$} \\
\hline 3.00 & 0.02 & 0.05 & 0.00026 & $\mathrm{ED}, \mathrm{ME}, \mathrm{FO}$ & [28] \\
\hline 1.00 & 0.04 & 0.04 & 0.00012 & ME & {$[28,29]$} \\
\hline 2.00 & 0.05 & 0.11 & 0.00157 & $M E, I D, L I$ & {$[28,29,46]$} \\
\hline 1.00 & 0.04 & 0.04 & 0.00023 & $\mathrm{ED}, \mathrm{ME}$ & {$[65,75]$} \\
\hline 2.60 & 0.09 & 0.23 & 0.00942 & $\mathrm{ED}, \mathrm{ME}, \mathrm{OR}, \mathrm{GR}, \mathrm{FO}$ & {$[27-29,46,59]$} \\
\hline 1.00 & 0.02 & 0.02 & 0.00003 & $\mathrm{FO}$ & {$[28]$} \\
\hline 1.00 & 0.05 & 0.05 & 0.00026 & $\mathrm{FO}$ & {$[29,40,62]$} \\
\hline 1.00 & 0.04 & 0.04 & 0.00023 & FO,EN & {$[27,62]$} \\
\hline 2.00 & 0.02 & 0.04 & 0.00012 & $\mathrm{FO}, \mathrm{EN}$ & [28] \\
\hline 1.00 & 0.04 & 0.04 & 0.00012 & $\mathrm{FO}$ & {$[57,62]$} \\
\hline 2.33 & 0.05 & 0.13 & 0.00244 & $\mathrm{ED}, \mathrm{ME}, \mathrm{FO}, \mathrm{GR}$ & {$[28,29,46]$} \\
\hline 2.00 & 0.05 & 0.11 & 0.00104 & $\mathrm{FO}, \mathrm{Fl}$ & [27-29] \\
\hline 3.71 & 0.13 & 0.46 & 0.03693 & $\mathrm{ED}, \mathrm{FI}, \mathrm{ME}, \mathrm{LI}, \mathrm{EN}, \mathrm{OT}, \mathrm{OR}$ & {$[27,29,45,46,62,64,65]$} \\
\hline 2.00 & 0.04 & 0.07 & 0.00070 & $\mathrm{ME}, \mathrm{FI}, \mathrm{EN}$ & {$[28,46]$} \\
\hline 1.00 & 0.05 & 0.05 & 0.00026 & ME & {$[28,29,46]$} \\
\hline 2.75 & 0.07 & 0.20 & 0.00510 & $\mathrm{ED}, \mathrm{ME}, \mathrm{PE}, \mathrm{ID}$ & {$[27-29,69]$} \\
\hline 1.20 & 0.09 & 0.11 & 0.00174 & $\mathrm{ME}, \mathrm{FO}$ & {$[27-29,58,46]$} \\
\hline 1.50 & 0.14 & 0.21 & 0.00835 & $\mathrm{ME}, \mathrm{FO}, \mathrm{GR}$ & {$[27-29,46,58,57,59,62]$} \\
\hline 1.20 & 0.09 & 0.11 & 0.00261 & $\mathrm{ME}, \mathrm{FO}, \mathrm{ED}$ & {$[28,46,57,65,70]$} \\
\hline 1.50 & 0.04 & 0.05 & 0.00035 & $\mathrm{ME}, \mathrm{FO}$ & {$[28,46]$} \\
\hline 1.00 & 0.09 & 0.09 & 0.00145 & $\mathrm{ME}, \mathrm{ED}$ & {$[28,46,69,70,76]$} \\
\hline
\end{tabular}




\section{Table 4 Ethnobotanical inventory and some quantitative indexes of useful wetland plants in China (Continued)}

\begin{tabular}{|c|c|c|c|c|c|c|}
\hline Pluchea indica (L.) Less. & 1.33 & 0.05 & 0.07 & 0.00070 & $\mathrm{ED}, \mathrm{ME}$ & {$[28,29,46]$} \\
\hline Poa acroleuca Steud. & 1.00 & 0.02 & 0.02 & 0.00003 & $\mathrm{FO}$ & [28] \\
\hline Pogonatherum crinitum Kunth & 1.33 & 0.05 & 0.07 & 0.00070 & $\mathrm{ME}, \mathrm{FO}$ & {$[28,29,46]$} \\
\hline Polygonum amphibium L. & 1.00 & 0.05 & 0.05 & 0.00026 & ME & {$[28,46,55]$} \\
\hline Polygonum aviculare L. & 1.50 & 0.14 & 0.21 & 0.01391 & $\mathrm{ED}, \mathrm{ME}, \mathrm{FO}, \mathrm{ID}, \mathrm{PE}$ & {$[28,29,46,57,58,67,70,74]$} \\
\hline Polygonum barbatum L. & 1.00 & 0.04 & 0.04 & 0.00012 & ME & {$[28,46]$} \\
\hline Polygonum capitatum Buch.-Ham. ex D. Don & 1.00 & 0.07 & 0.07 & 0.00046 & ME & {$[28,29,46,58]$} \\
\hline Polygonum chinense $\mathrm{L}$. & 1.00 & 0.05 & 0.05 & 0.00026 & ME & {$[28,29,46]$} \\
\hline Polygonum excurrens Steward & 1.00 & 0.02 & 0.02 & 0.00003 & ME & {$[55]$} \\
\hline Polygonum hydropiper L. & 1.41 & 0.30 & 0.43 & 0.07096 & ME,ED,FO,OT,PE,ID & {$[28,29,45,46,50-53,55,57-59,62,63,67,70,75]$} \\
\hline Polygonum japonicum Meisn. & 1.17 & 0.11 & 0.13 & 0.00244 & $M E, P E$ & {$[27-29,46,55,58]$} \\
\hline Polygonum jucundum Meisn. & 1.00 & 0.04 & 0.04 & 0.00023 & $M E, P E$ & {$[55,58]$} \\
\hline Polygonum kawagoeanum Makino & 1.00 & 0.02 & 0.02 & 0.00003 & ME & {$[58]$} \\
\hline Polygonum lapathifolium L. & 2.13 & 0.14 & 0.30 & 0.03154 & ED,ME,FO,PE,LI,ID,EN,GR & {$[27,46,50,55,57,58,62,67]$} \\
\hline Polygonum lapathifolium var. salicifolium Sibth. & 2.50 & 0.04 & 0.09 & 0.00116 & $M E, P E, L I, I D$ & {$[55,58]$} \\
\hline Polygonum longisetum var. rotundatum A. J. Li & 1.25 & 0.07 & 0.09 & 0.00116 & $M E, I D$ & {$[27,46,55,58]$} \\
\hline Polygonum macranthum Meisn. & 1.00 & 0.04 & 0.04 & 0.00012 & ME & {$[55,58]$} \\
\hline Polygonum nepalense Meisn. & 1.00 & 0.05 & 0.05 & 0.00026 & ME & {$[28,46,58]$} \\
\hline Polygonum orientale L. & 2.60 & 0.18 & 0.46 & 0.05276 & $\mathrm{ED}, \mathrm{ME}, \mathrm{FO}, \mathrm{PE}, \mathrm{LI}, \mathrm{OR}, \mathrm{ID}$ & {$[27-29,45,46,55,57-59,62]$} \\
\hline Polygonum perfoliatum L. & 2.50 & 0.07 & 0.18 & 0.00348 & ME,PE,ID & {$[27,28,46,58]$} \\
\hline Polygonum persicaria $\mathrm{L}$. & 1.33 & 0.05 & 0.07 & 0.00104 & $\mathrm{ME}, \mathrm{FO}, \mathrm{PE}$ & {$[28,58,67]$} \\
\hline Polygonum posumbu Buch.-Ham. ex D. Don & 1.00 & 0.02 & 0.02 & 0.00003 & ME & {$[58]$} \\
\hline Polygonum sibiricum Laxm. & 2.00 & 0.02 & 0.04 & 0.00012 & $\mathrm{ME}, \mathrm{FO}$ & [28] \\
\hline Polygonum sieboldii Meisn. & 1.00 & 0.05 & 0.05 & 0.00026 & ME & {$[28,29,46]$} \\
\hline Polygonum taquetii H. Lév. & 1.00 & 0.02 & 0.02 & 0.00003 & ME & {$[58]$} \\
\hline Polygonum thunbergii Siebold \& Zucc. & 2.00 & 0.05 & 0.11 & 0.00209 & $\mathrm{ED}, \mathrm{ME}, \mathrm{FO}, \mathrm{ID}$ & {$[28,46,58]$} \\
\hline Polygonum viscosum Buch.Ham. ex D. Don & 1.67 & 0.05 & 0.09 & 0.00130 & $E D, M E, I D$ & {$[28,58,63]$} \\
\hline Potamogeton crispus L. & 2.00 & 0.11 & 0.21 & 0.00835 & $\mathrm{ED}, \mathrm{ME}, \mathrm{FO}, \mathrm{GR}$ & {$[27,29,35,46,62,64]$} \\
\hline Potamogeton cristatus Regel \& Maack & 1.00 & 0.04 & 0.04 & 0.00023 & $\mathrm{ME}, \mathrm{FO}$ & {$[46,62]$} \\
\hline Potamogeton distinctus A. Benn. & 1.33 & 0.05 & 0.07 & 0.00070 & $F O, G R$ & {$[27,28,62]$} \\
\hline Potamogeton lucens L. & 1.00 & 0.04 & 0.04 & 0.00012 & $G R$ & {$[27,62]$} \\
\hline Potamogeton maackianus A. Benn. & 1.00 & 0.02 & 0.02 & 0.00003 & $\mathrm{FO}$ & {$[62]$} \\
\hline Potamogeton malaianus Miq. & 2.00 & 0.05 & 0.11 & 0.00104 & $F O, G R$ & {$[27,28,62]$} \\
\hline Potamogeton natans $\mathrm{L}$. & 1.00 & 0.05 & 0.05 & 0.00078 & $\mathrm{ME}, \mathrm{FO}, \mathrm{GR}$ & {$[46,54,64]$} \\
\hline Potamogeton octandrus Poir. & 1.00 & 0.02 & 0.02 & 0.00003 & $\mathrm{FO}$ & {$[62]$} \\
\hline Potamogeton oxyphyllus Miq. & 1.00 & 0.02 & 0.02 & 0.00003 & $\mathrm{FO}$ & {$[62]$} \\
\hline Potamogeton pectinatus $\mathrm{L}$. & 1.60 & 0.09 & 0.14 & 0.00348 & $\mathrm{ME}, \mathrm{FO}, \mathrm{GR}$ & {$[27-29,46,62]$} \\
\hline Potamogeton perfoliatus $\mathrm{L}$. & 1.00 & 0.02 & 0.02 & 0.00003 & ME & [46] \\
\hline Potamogeton pusillus $\mathrm{L}$. & 2.00 & 0.04 & 0.07 & 0.00046 & $F O, G R$ & {$[27,62]$} \\
\hline Potentilla anserina $\mathrm{L}$. & 2.17 & 0.11 & 0.23 & 0.01357 & $\mathrm{ED}, \mathrm{ME}, \mathrm{FO}, \mathrm{ID}, \mathrm{OT}, \mathrm{LI}$ & {$[28,29,46,70,69,79]$} \\
\hline Potentilla discolor Bunge & 1.33 & 0.05 & 0.07 & 0.00070 & $\mathrm{ED}, \mathrm{ME}$ & {$[28,29,46]$} \\
\hline Potentilla flagellaris D. F. K. Schltdl. & 2.00 & 0.04 & 0.07 & 0.00093 & $\mathrm{ED}, \mathrm{ME}, \mathrm{FO}, \mathrm{GR}$ & {$[28,29]$} \\
\hline Potentilla kleiniana Wight \& Arn. & 1.00 & 0.05 & 0.05 & 0.00026 & ME & {$[28,29,46]$} \\
\hline Potentilla reptans $\mathrm{L}$. & 1.50 & 0.04 & 0.05 & 0.00035 & $\mathrm{ED}, \mathrm{ME}$ & {$[28,46]$} \\
\hline Prunella vulgaris $\mathrm{L}$. & 1.00 & 0.05 & 0.05 & 0.00026 & ME & {$[28,29,46]$} \\
\hline
\end{tabular}




\section{Table 4 Ethnobotanical inventory and some quantitative indexes of useful wetland plants in China (Continued)}

\begin{tabular}{|c|c|c|c|c|c|c|}
\hline Pseudoraphis sordida (Thwaites) S. M. Phillips \& S. L. Chen & 1.00 & 0.04 & 0.04 & 0.00012 & $\mathrm{FO}$ & {$[27,29]$} \\
\hline Ranunculus cantoniensis DC. & 1.00 & 0.07 & 0.07 & 0.00046 & ME & {$[28,29,46,58]$} \\
\hline Ranunculus chinensis Bunge & 1.17 & 0.11 & 0.13 & 0.00244 & ME,PE & {$[27-29,46,58,68]$} \\
\hline Ranunculus japonicus Thunb. & 1.17 & 0.11 & 0.13 & 0.00244 & ME,PE & {$[27-29,46,58,74]$} \\
\hline Ranunculus sceleratus L. & 1.00 & 0.09 & 0.09 & 0.00072 & ME & {$[28,29,46,58,59]$} \\
\hline Ranunculus sieboldii Miq. & 1.00 & 0.07 & 0.07 & 0.00046 & ME & {$[27-29,46]$} \\
\hline Ranunculus ternatus Thunb. & 1.00 & 0.09 & 0.09 & 0.00072 & ME & {$[27-29,46,58]$} \\
\hline Reynoutria japonica Houtt. & 2.50 & 0.04 & 0.09 & 0.00116 & $\mathrm{ED}, \mathrm{ME}, \mathrm{PE}, \mathrm{ID}$ & {$[28,46]$} \\
\hline Roegneria ciliaris (Trin.) Nevski & 1.00 & 0.05 & 0.05 & 0.00026 & $\mathrm{FO}$ & {$[28,29,57]$} \\
\hline Rorippa dubia (Pers.) Hara & 2.50 & 0.04 & 0.09 & 0.00116 & $\mathrm{ED}, \mathrm{ME}, \mathrm{FO}, \mathrm{ID}$ & {$[28,29]$} \\
\hline Rorippa globosa (Turcz.) Hayek & 1.60 & 0.09 & 0.14 & 0.00348 & $\mathrm{ED}, \mathrm{FO}, \mathrm{ID}$ & {$[27,28,58,62,67]$} \\
\hline Rorippa islandica (Oeder) Borbás & 3.33 & 0.05 & 0.18 & 0.00348 & $\mathrm{ED}, \mathrm{ME}, \mathrm{FO}, \mathrm{ID}$ & {$[27,28,45]$} \\
\hline Rotala indica Koehne & 1.00 & 0.07 & 0.07 & 0.00139 & $\mathrm{ED}, \mathrm{ME}, \mathrm{FO}$ & {$[27,28,63,62]$} \\
\hline Rotala rotundifolia (Buch.-Ham. ex Roxb.) Koehne & 1.00 & 0.07 & 0.07 & 0.00093 & $\mathrm{ME}, \mathrm{FO}$ & {$[46,75,29,62]$} \\
\hline Rumex acetosa L. & 3.17 & 0.11 & 0.34 & 0.01652 & $E D, M E, F O, P E, I D$ & {$[27-29,46,58,65]$} \\
\hline Rumex crispus L. & 1.00 & 0.09 & 0.09 & 0.00145 & $\mathrm{ED}, \mathrm{ME}$ & {$[28,30,46,67,79]$} \\
\hline Rumex dentatus $\mathrm{L}$. & 1.75 & 0.07 & 0.13 & 0.00244 & $\mathrm{ME}, \mathrm{FO}, \mathrm{PE}$ & {$[27,28,46,62]$} \\
\hline Rumex japonicus Houtt. & 2.60 & 0.09 & 0.23 & 0.00942 & $\mathrm{ED}, \mathrm{ME}, \mathrm{FO}, \mathrm{ID}, \mathrm{LI}$ & {$[28,29,46,57,58]$} \\
\hline Rumex maritimus L. & 1.33 & 0.05 & 0.07 & 0.00070 & $\mathrm{ME}, \mathrm{FO}$ & {$[28,29,63]$} \\
\hline Rumex nepalensis Spreng. & 1.25 & 0.07 & 0.09 & 0.00116 & $M E, I D$ & {$[28,29,46,68]$} \\
\hline Rumex patientia L. & 2.50 & 0.04 & 0.09 & 0.00116 & $\mathrm{ED}, \mathrm{ME}, \mathrm{ID}, \mathrm{LI}$ & {$[28,46]$} \\
\hline Rungia chinensis Benth. & 1.00 & 0.02 & 0.02 & 0.00003 & ME & {$[28]$} \\
\hline Saccharum spontaneum L. & 3.00 & 0.05 & 0.16 & 0.00313 & $\mathrm{FI}, \mathrm{FO}, \mathrm{OT}, \mathrm{EN}$ & {$[27-29]$} \\
\hline Sacciolepis indica (L.) Chase & 1.00 & 0.04 & 0.04 & 0.00012 & $\mathrm{FO}$ & {$[27,28]$} \\
\hline Sacciolepis myosuroides (R. Br.) A.Camus & 1.00 & 0.04 & 0.04 & 0.00012 & $\mathrm{FO}$ & {$[28,29]$} \\
\hline Sagina japonica (Sw. ex Steud.) Ohwi & 1.00 & 0.07 & 0.07 & 0.00046 & ME & {$[27,28,46,58]$} \\
\hline Sagittaria pygmaea Miq. & 1.25 & 0.07 & 0.09 & 0.00174 & $\mathrm{ME}, \mathrm{FO}, \mathrm{GR}$ & {$[27-29,46]$} \\
\hline Sagittaria trifolia L. & 1.88 & 0.14 & 0.27 & 0.01739 & ED,ME,FO,LI,OR & {$[27-29,45,46,63,65,64]$} \\
\hline Salicornia europaea L. & 2.50 & 0.04 & 0.09 & 0.00087 & $M E, I D, E N$ & {$[27,28]$} \\
\hline Salvia plebeia R. Br. & 1.00 & 0.07 & 0.07 & 0.00046 & ME & {$[27-29,46]$} \\
\hline Salvinia natans (L.) All. & 1.89 & 0.16 & 0.30 & 0.01331 & $\mathrm{ME}, \mathrm{FO}, \mathrm{GR}$ & {$[27-30,46,57,58,62,64]$} \\
\hline Saururus chinensis Hort. ex Loudon & 1.33 & 0.05 & 0.07 & 0.00070 & $M E, G R$ & {$[28,29,46]$} \\
\hline Scirpus juncoides Roxb. & 1.50 & 0.04 & 0.05 & 0.00035 & $\mathrm{ME}, \mathrm{Fl}$ & {$[28,46]$} \\
\hline Scirpus planiculmis F.Schmidt & 2.75 & 0.07 & 0.20 & 0.00765 & $\mathrm{ED}, \mathrm{ME}, \mathrm{FI}, \mathrm{FO}, \mathrm{LI} \mathrm{L} \mathrm{EN}$ & {$[28,57,62,67]$} \\
\hline Scirpus tabernaemontani Salzm. ex Ball & 2.50 & 0.11 & 0.27 & 0.01304 & $\mathrm{ME}, \mathrm{Fl}, \mathrm{FO}, \mathrm{EN}, \mathrm{OR}$ & {$[27,28,45,57,64,65]$} \\
\hline Scirpus triangulatus Roxb. & 1.40 & 0.09 & 0.13 & 0.00304 & $\mathrm{ME}, \mathrm{FO}, \mathrm{FI}$ & {$[28,29,45,46,67]$} \\
\hline Scirpus triqueter $\mathrm{L}$. & 1.43 & 0.13 & 0.18 & 0.00406 & $\mathrm{Fl}, \mathrm{FO}$ & {$[27-29,45,57,62,64]$} \\
\hline Scirpus wallichii Nees & 1.00 & 0.02 & 0.02 & 0.00003 & ME & [28] \\
\hline Scirpus yagara Ohwi & 2.57 & 0.13 & 0.32 & 0.01826 & $\mathrm{ME}, \mathrm{FI}, \mathrm{FO}, \mathrm{ID}, \mathrm{LI}$ & {$[27,28,45,46,59,62,64]$} \\
\hline Scrophularia ningpoensis Hemsl. & 1.00 & 0.05 & 0.05 & 0.00026 & ME & {$[28,29,46]$} \\
\hline Sesbania cannabina (Retz.) Poir. & 2.67 & 0.05 & 0.14 & 0.00417 & $\mathrm{ME}, \mathrm{FI}, \mathrm{FO}, \mathrm{GR}, \mathrm{EN}, \mathrm{ID}$ & {$[28,29,46]$} \\
\hline Sinosenecio oldhamianus (Maxim.) B. Nord. & 2.00 & 0.04 & 0.07 & 0.00046 & $\mathrm{FO}, \mathrm{GR}$ & {$[28,57]$} \\
\hline Sium suave Walter & 1.00 & 0.07 & 0.07 & 0.00093 & $\mathrm{ME}, \mathrm{FO}$ & {$[28,29,46,64]$} \\
\hline Solanum torvum Sw. & 1.33 & 0.05 & 0.07 & 0.00070 & $\mathrm{ED}, \mathrm{ME}$ & {$[28,29,46]$} \\
\hline Sparganium stoloniferum (Graebn.) Buch.-Ham. ex Juz. & 1.78 & 0.16 & 0.29 & 0.02505 & $\mathrm{ME}, \mathrm{OR}, \mathrm{FO}, \mathrm{Fl}, \mathrm{GR}, \mathrm{OT}$ & {$[27-29,45,46,57,59,62,64]$} \\
\hline
\end{tabular}




\section{Table 4 Ethnobotanical inventory and some quantitative indexes of useful wetland plants in China (Continued)}

\begin{tabular}{|c|c|c|c|c|c|c|}
\hline Spilanthes paniculata Wall. & 1.00 & 0.05 & 0.05 & 0.00026 & ME & {$[28,29,46]$} \\
\hline Spirodela polyrhiza (L.) Schleid. & 1.50 & 0.18 & 0.27 & 0.01304 & $\mathrm{ME}, \mathrm{FO}, \mathrm{GR}$ & {$[27-29,46-48,59,63,64,62]$} \\
\hline Stachys adulterina Hemsl. & 1.75 & 0.07 & 0.13 & 0.00162 & $\mathrm{ED}, \mathrm{ME}$ & {$[27,46,58,61]$} \\
\hline Stachys chinensis Bunge ex Benth. & 1.00 & 0.04 & 0.04 & 0.00012 & ME & {$[46,64]$} \\
\hline Stachys japonica Miq. & 1.75 & 0.07 & 0.13 & 0.00325 & $\mathrm{ED}, \mathrm{ME}, \mathrm{ID}, \mathrm{LI}$ & {$[27-29,58]$} \\
\hline Stachys oblongifolia Wall. & 1.33 & 0.05 & 0.07 & 0.00070 & $\mathrm{ME}, \mathrm{FO}$ & {$[28,29,46]$} \\
\hline Stellaria uliginosa Murray & 1.00 & 0.04 & 0.04 & 0.00023 & $\mathrm{ME}, \mathrm{FO}$ & {$[28,46]$} \\
\hline Stephania japonica (Thunb.) Miers & 1.00 & 0.09 & 0.09 & 0.00072 & ME & {$[27-29,46,80]$} \\
\hline Suaeda glauca Bunge & 1.60 & 0.09 & 0.14 & 0.00464 & $\mathrm{ED}, \mathrm{ME}, \mathrm{ID}, \mathrm{OT}$ & {$[27-29,46,70]$} \\
\hline Suaeda salsa Pall. & 2.00 & 0.05 & 0.11 & 0.00209 & $\mathrm{ED}, \mathrm{FO}, \mathrm{GR}, \mathrm{ID}$ & {$[27,29,70]$} \\
\hline Thalictrum simplex L. & 1.00 & 0.05 & 0.05 & 0.00026 & $\mathrm{ME}$ & {$[28,29,46]$} \\
\hline Trapa bicornis L. f. & 1.67 & 0.16 & 0.27 & 0.01957 & $\mathrm{ED}, \mathrm{ME}, \mathrm{FO}, \mathrm{ID}, \mathrm{LI}$ & {$[27,29,45,46,57,58,62,65,73]$} \\
\hline Trapa bispinosa Roxb. & 2.33 & 0.05 & 0.13 & 0.00244 & $\mathrm{ED}, \mathrm{ME}, \mathrm{FO}, \mathrm{LI}$ & {$[28,29,46]$} \\
\hline Trapa incisa Siebold \& Zucc. & 1.50 & 0.07 & 0.11 & 0.00278 & $\mathrm{ED}, \mathrm{ME}, \mathrm{FO}, \mathrm{ID}$ & {$[27,29,46,65]$} \\
\hline Trapa maximowiczii Korsh. & 1.50 & 0.04 & 0.05 & 0.00035 & $E D, L I$ & {$[28,29]$} \\
\hline Trifolium pratense $\mathrm{L}$. & 2.50 & 0.04 & 0.09 & 0.00116 & $M E, F O, G R, I D$ & {$[28,46]$} \\
\hline Typha angustata Bory \& Chaub. & 1.50 & 0.04 & 0.05 & 0.00035 & $\mathrm{ME}, \mathrm{Fl}$ & {$[28,46]$} \\
\hline Typha angustifolia L. & 1.63 & 0.14 & 0.23 & 0.00904 & $\mathrm{ED}, \mathrm{ME}, \mathrm{FI}$ & {$[27-29,45,46,64,65,67]$} \\
\hline Typha latifolia L. & 2.17 & 0.11 & 0.23 & 0.00904 & $\mathrm{FI}, \mathrm{ME}, \mathrm{ED}, \mathrm{OR}$ & {$[28,29,46,64,65,70]$} \\
\hline Typha orientalis C. Presl & 2.13 & 0.14 & 0.30 & 0.01577 & $M E, F I, E D, O R$ & {$[27-29,33,34,46,59,64]$} \\
\hline Typhonium giganteum Engl. & 1.00 & 0.05 & 0.05 & 0.00026 & ME & {$[28,29,46]$} \\
\hline Urtica angustifolia Fisch. ex Hornem. & 2.33 & 0.05 & 0.13 & 0.00244 & $\mathrm{ED}, \mathrm{ME}, \mathrm{FI}, \mathrm{ID}$ & {$[28,29,46]$} \\
\hline Utricularia aurea Lour. & 1.00 & 0.04 & 0.04 & 0.00023 & $\mathrm{FO}, \mathrm{OR}$ & {$[58,60]$} \\
\hline Utricularia vulgaris L. & 1.00 & 0.02 & 0.02 & 0.00003 & $\mathrm{FO}$ & [64] \\
\hline Valeriana flaccidissima Maxim. & 1.00 & 0.02 & 0.02 & 0.00003 & ME & {$[28]$} \\
\hline Valeriana officinalis $\mathrm{L}$. & 1.00 & 0.05 & 0.05 & 0.00026 & ME & {$[28,29,46]$} \\
\hline Vallisneria natans (Lour.) H. Hara & 1.33 & 0.05 & 0.07 & 0.00070 & $\mathrm{ME}, \mathrm{FO}$ & {$[27,28,46]$} \\
\hline Verbena officinalis $\mathrm{L}$. & 1.00 & 0.09 & 0.09 & 0.00072 & ME & {$[28,29,46,68,78]$} \\
\hline Veronica anagallis-aquatica L. & 1.33 & 0.05 & 0.07 & 0.00070 & $\mathrm{ED}, \mathrm{ME}$ & {$[28,29,46]$} \\
\hline Veronica undulata Wall. & 1.00 & 0.04 & 0.04 & 0.00012 & $\mathrm{ME}$ & {$[28,29]$} \\
\hline Vicia bungei Ohwi & 2.00 & 0.04 & 0.07 & 0.00046 & $F O, G R$ & {$[28,57]$} \\
\hline Viola grypoceras A.Gray & 1.00 & 0.05 & 0.05 & 0.00026 & ME & {$[27-29]$} \\
\hline Viola inconspicua Blume & 1.00 & 0.09 & 0.09 & 0.00072 & ME & {$[27-29,46,58]$} \\
\hline Wolffia arrhiza (L.) Wimm. & 1.50 & 0.07 & 0.11 & 0.00209 & $\mathrm{ED}, \mathrm{FO}, \mathrm{GR}$ & {$[27-29,48]$} \\
\hline Zantedeschia aethiopica (L.) Spreng. & 1.00 & 0.04 & 0.04 & 0.00023 & OR,OT & {$[27,29]$} \\
\hline Zizania latifolia Turcz. & 3.33 & 0.16 & 0.54 & 0.06262 & $\mathrm{ED}, \mathrm{ME}, \mathrm{FI}, \mathrm{FO}, \mathrm{OT}, \mathrm{ID}, \mathrm{GR}, \mathrm{OR}$ & {$[27,29,39,45,46,57,62,64,65]$} \\
\hline
\end{tabular}

UV: Use value; RFC: Relative Frequency of Citation; Cl: Cultural Importance Index; CV: Cultural Value Index.

Wetland plants provide people with many types of products valuable for subsistence living. The wealth of traditional knowledge that has accumulated about the uses of wetland plants is a reflection of the close relationships traditionally existing between people and their local environments, in this case specifically relating to wetlands. Much of this knowledge is disappearing today along with the loss of traditional lifestyles and retreat of wetlands. Systematic ethnobotanical surveys of traditional knowledge relating to wetlands are therefore needed, while such knowledge still exists.

\section{Comparison of some quantitative indexes}

An increasing number of papers have appeared over recent years discussing the use of quantitative methods in ethnobotanical research $[101,102]$. In particular, many new parameters have been suggested for evaluating the cultural importance or significance of plants and 


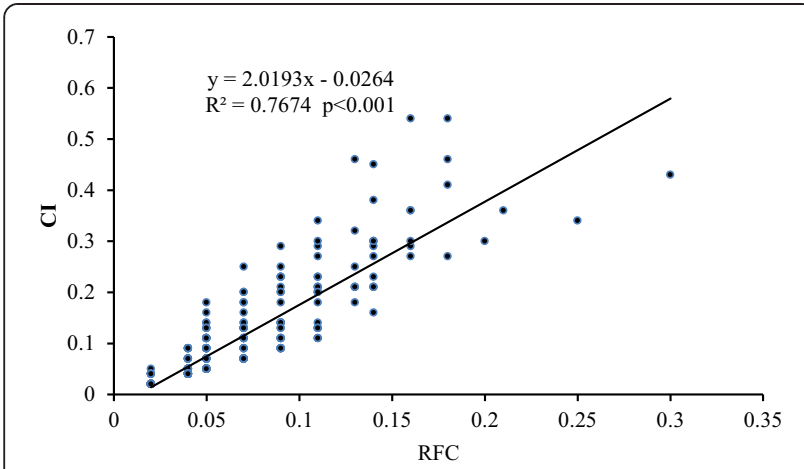

Figure $\mathbf{2}$ The correlation between Relative Frequency of Citation (RFC) and Cultural Importance (CI).

determine information consensus between informants [24-26,103]. The use of such indexes can not only advance the development of ethnobotany, but can also make it possible to compare results between different regions or cultural groups, as well as undertaking metaanalyses.

Use Value (UV) is one of the most frequently used indexes for evaluating 'the relative usefulness of plants to people' $[23,24,83,84]$. It has been successfully applied in many contexts [104-108]. With respect to an analysis of the literature, such as that here, UV reflects not only the number of uses made of a plant as well as the number of literature sources mentioning it. So a plant with high UV value does not necessarily mean that it has multiple uses nor that it is necessarily mentioned in many publications, as we have discussed in an earlier paper [109]. To illustrate this point, three species (Najas graminea, Potamogeton pusillus and Monochoria korsakowii) were all found to have UV $=2$ in the present study, but actually the first two of these are only mentioned in two literature sources with two uses in each case, while the third is mentioned in 8 sources but only for one type of use. Among those plants with UV =1, they have the same total numbers of different uses recorded in the

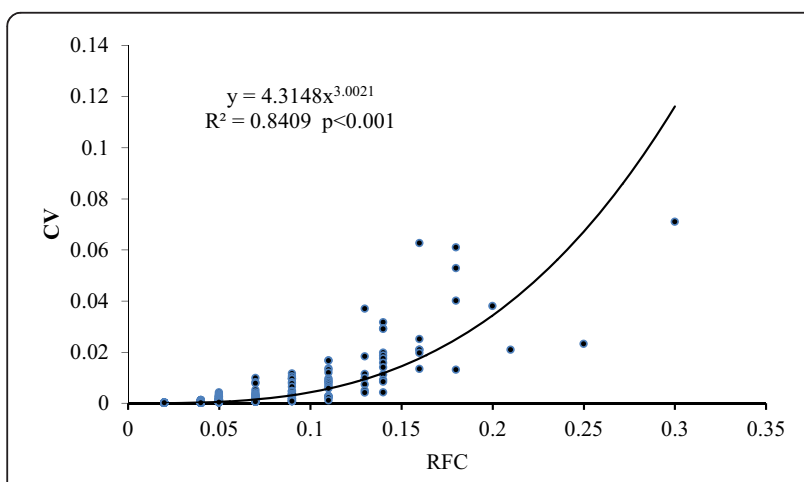

Figure 3 The correlation between Relative Frequency of Citation (RFC) and Cultural Value Index (CV).

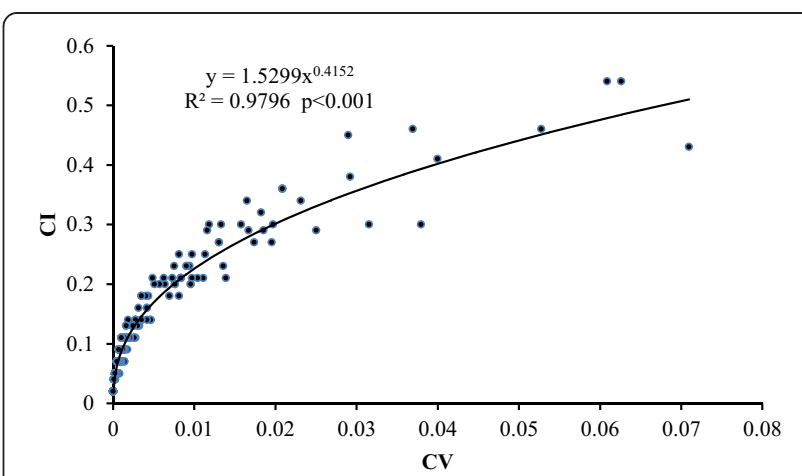

Figure 4 The correlation between Cultural Value Index (CV) and Cultural Importance (CI).

literature and the numbers of literature recording these uses. Although their UV values are the lowest, it does not mean that they have few uses. However, some plants with higher UV values are indeed versatile, such as $P$. australis, $Z$. latifolia, and $N$. tetragona. These plants have a common feature: mentioned by a higher number of literature. So the UV value in a literature study may give us a bias. When using UV index to evaluate a plant, we should use the number of the literature recorded it for reference.

According the formula used for calculating FUV, we can find that FUV depends on the UV of species in a family. So FUV has a similar shortcoming to UV.

Compared with UV, RFC, CI and CV have considered more factors that may lead to a bias. RFC is as same as $\% \mathrm{P}$ designed in one of our previous papers [109]. Although it has considered the number of the literature which mentions a given species and the total number of literature concerned in the study, it does not take into account the number of uses mentioned in the literature. It just reflects the frequency of a species mentioned by the literature. There are significantly positive correlations between RFC and CI $\left(R^{2}=0.767, p<0.001\right)$ and CV $\left(R^{2}=0.841, p<0.001\right)$, respectively (Figure 2 and 3 ). Because RFC does not consider the number of uses, it will not show the difference of the importance and use values between species. Compared with RFC, $\mathrm{CI}$ and $\mathrm{CV}$ are two more comprehensive indexes. They consider not only the frequency cited by the literature, but also the number of uses recorded in the literature. There is a significant correlation between $\mathrm{CI}$ and $\mathrm{CV}\left(\mathrm{R}^{2}=0.980\right.$, $\mathrm{p}<0.001$ ). The species with higher CI values often have higher $\mathrm{CV}$, such as $P$. hydropiper and $Z$. latifolia. Compared with $\mathrm{CI}, \mathrm{CV}$ is more sensitive to the information recorded in the literature and is more effective to show the differences of use value and frequency being cited in the literature between species.

In fact, many indexes used in quantitative ethnobotany are related to the use categories. The method of use type 
classification will affect greatly the calculation results. However, it is often very difficult to produce a perfect scheme of use categories which could be closer to the truth. What can we do is to try our best to make the use categories more reasonable.

\section{Conclusion}

The wide distribution of many species of wetland plants $[110,111]$ makes it possible to gain a general picture of the uses made of such plants on a macro-scale. A principal conclusion from the present study is that the biggest uses of wetland species, in terms of the number of citations in the literature, are for medicine, food and fodder. We conclude that it is whether or not particular species are growing locally that is a major determinant over whether people actually use them. Cultural Value (CV) and Cultural Importance $(\mathrm{CI})$ are judged to be the most useful quantitative indices for providing measures of the relative importance and usefulness of wetland species, based on analyses of citations in literature that is not specifically ethnobotanical. However, such publications cannot provide detailed information about relationships between wetland plants and people, such as details of the ways in which people use and manage them. China is rich in both wetlands and traditional knowledge of wetland plants, but both wetlands and traditional knowledge are rapidly being lost. Traditional knowledge about wetland plants has much to offer for modern needs, such as the sustainable use of wetland plants, conservation and industrial development. We therefore conclude that there is a great need for detailed systematic ethnobotanical studies on wetland plants to be undertaken as a matter of urgency.

\section{Competing interests}

The authors declare that they have no competing interests.

\section{Authors' contributions}

$\mathrm{YZ}$ and $\mathrm{HH}$ conceived of the study, participated in its design, data collection and analysis, and helped to draft the manuscript. HLX participated in data analysis and enrichment of manuscript. HC and FW participated in data collection, analysis and revision of the manuscript. All authors read and approved the final manuscript.

\section{Acknowledgements}

Many thanks to Dr. Alan Hamilton for assisting with the English. The authors also thank two anonymous reviewers for their valuable comments for the paper. This study was financially supported by grants from the National Natural Science Foundation of China (31170299 and 31370355).

Received: 23 May 2014 Accepted: 22 September 2014

Published: 15 October 2014

\section{References}

1. Bekalo TH, Woodmatas SD, Woldemariam ZA: An ethnobotanical study of medicinal plants used by local people in the lowlands of Konta Special Woreda, southern nations, nationalities and peoples regional state, Ethiopia. J Ethnobiol Ethnomed 2009, 5:26.

2. Patra R: Vaastu Shastra: towards sustainable development. Sust Dev 2009, 17(4):244-256
3. Rana MMP: Urbanization and sustainability: challenges and strategies for sustainable urban development in Bangladesh. Environ Dev Sustain 2011, 13(1):237-256.

4. Huai $\mathrm{H}, \mathrm{Xu}$ J: Indigenous knowledge: an inexhaustible "information bank" for toxin research. Toxicon 2000, 38(6):745-746.

5. Ramirez CR: Ethnobotany and the loss of traditional knowledge in the 21st Century. Ethnobot Res Appl 2007, 5:245-247.

6. Volpato G, Godínez D, Beyra A: Migration and ethnobotanical practices: The case of tifey among Haitian immigrants in Cuba. Hum Ecol 2009, 37(1):43-53.

7. Cetinkale SG, Acıksöz S: Sustainability of traditional pattern in urban landscape: The case of Bartin. J Agric Sci 2007, 13(2):81-88.

8. Wu T, Petriello MA: Culture and biodiversity losses linked. Science 2011, 331(6013):30-31.

9. Denny P: Biodiversity and wetlands. Wetl Ecol Manag 1994, 3(1):55-61.

10. Schuyt KD: Economic consequences of wetland degradation for local populations in Africa. Ecol Econ 2005, 53:177-190.

11. Jain A, Sundriyal M, Roshnibala S, Kotoky R, Kanjilal PB, Singh HB, Sundriyal RC: Dietary use and conservation concern of edible wetland plants at Indo-Burma hotspot: a case study from Northeast India. J Ethnobiol Ethnomed 2011, 7:29

12. Panda A, Misra MK: Ethnomedicinal survey of some wetland plants of South Orissa and their conservation. Indian J Tradit Know 2011, 10(2):296-303.

13. Gichuki J, Guebas FD, Mugo J, Rabuor CO, Triest L, Dehairs F: Species inventory and the local uses of the plants and fishes of the Lower Sondu Miriu wetland of Lake Victoria, Kenya. Hydrobiologia 2001, 458:99-106.

14. Ambastha K, Hussain SA, Badola R: Resource dependence and attitudes of local people toward conservation of Kabartal wetland: a case study from the Indo-Gangetic plains. Wetl Ecol Manag 2007, 15(4):287-302.

15. Ehrenfeld JG: Exotic invasive species in urban wetlands: environmental correlates and implications for wetland management. J Appl Ecol 2008, 45(4):1160-1169.

16. Zheng $X$, Li C, Huang G, Yang Z: Research progress in effects of urbanization on wetland ecosystem in watershed. Wet/ Sci 2008, 6(1):87-96.

17. Vallet J, Daniel H, Beaujouan V, Rozé F: Plant species response to urbanization: comparison of isolated woodland patches in two cities of North-Western France. Landscape Ecol 2008, 23(10):1205-1217.

18. Vermonden K, Leuven RSEW, van der Velde G, Hendriks A, van Katwijk MM, Roelofs JGM, Lucassen ECHET, Pedersen O, Sand-Jensen K: Species pool versus site limitations of macrophytes in urban waters. Aquat Sci 2010, 72(3):379-389.

19. McKinney ML: Effects of urbanization on species richness: a review of plants and animals. Urban Ecosyst 2008, 11:161-176.

20. Lei $K$, Zhang M: The wetland resources in China and the conservation advices. Wet/ Sci 2005, 3(2):81-86.

21. Albuquerque UP, Medeiros PM: Systematic reviews and meta-analysis applied to ethnobiological research. Ethnobio/ Conserv 2012, 1:6.

22. Bletter N: A quantitative synthesis of the medicinal ethnobotany of the Malinké of Mali and the Asháninka of Peru, with a new theoretical framework. J Ethnobiol Ethnomed 2007, 3:36.

23. Molares S, Ladio A: Ethnobotanical review of the Mapuche medicinal flora: use patterns on a regional scale. J Ethnopharmacol 2009, 122(2):251-260.

24. Tardío J, Pardo-de-Santayana M: Cultural importance indices: a comparative analysis based on the useful wild plants of Southern Cantabria (Northern Spain). Econ Bot 2008, 62(1):24-39.

25. de Medeiros PM, Ladio AH, Albuquerque UP: Patterns of medicinal plant use by inhabitants of Brazilian urban and rural areas: A macroscale investigation based on available literature. J Ethnopharmacol 2013, 150(2):729-746.

26. Reyes-García V, Huanca T, Vadez V, Leonard W, Wilkie D: Cultural, practical, and economic value of wild plants: a quantitative study in the Bolivian Amazon. Econ Bot 2006, 60(1):62-74.

27. Jiangsu Institute of Botany: Flora of Jiangsu. Nanjing: Jiangsu People's Publishing Ltd; 1977 [江苏植物研究所编，江苏植物志，江苏人民出版 社: 南京, 1977].

28. Li Y: Weed Flora of China. Beijing: China Agriculture Press; 1998 [李扬汉, 中 国杂草志, 中国农业出版社: 北京, 1998].

29. Editorial Committee of Flora of China: Flora of China. Beijing: Science Press; 1959_ 2004 [中国植物志编委会, 中国植物志, 科学出版社: 北京, 1959-2004].

30. Liu G, Li J, Shan L, Wang Q: Salvinia natans-—Green foods for poultry (家禽的绿色食粮-槐叶萍). Heilongjiang Anim Sci Vet Med 1999, 2:14-15. 
31. Jiao B, Gu R, Zhang X: Green manure. Beijing: China Agriculture Press; 1986 [中国绿肥，农业出版社: 北京, 1986].

32. Wang S: Use of Azolla imbricata in agriculture, China(满江红在我国农业 上的利用). Chin J Soil Sci 1980, 6:14.

33. Chinese Pharmacopoeia Commission: Pharmacopoeia of The People's Republic of China. Beijing: China Medical Science Press; 2010 [国家药典委员 会，中华人民共和国药典，中国医药科技出版社：北京，2010].

34. Yu H, Du L, Liu X: Integrated utilization of Typha orientalis (香蒲的综合利 用). Mod Chin Med 2007, 9(9):31-34.

35. Hou C, Guo G, Sun X: Value, harm and control measures of Potamogeton crispus (菹草的作用, 危害及预防措施). JiLin Agric 2011, 7:229.

36. Wu W: Arundo donax - a high quality raw material for paper-making (芦竹一一种高产优质的造纸原料). Tianjin Paper Making 1993, 4:28-29.

37. Hu X: Arundo donax is a good species of greening in waterbody rich areas (水网地区绿化好品种-芦竹). Jiangsu Greening 1996, 1:31.

38. Zhang J: Arundo donax var. versicolor, a new resource of aquatic species for greening (水生绿化植物新品种一花叶芦竹). New Rural Technol 2008, 9:33.

39. Zhai C, Sun G, Lu Z, Jiang Z, Zhang X: On Chinese Zizania L. resources and their utilization value (中国菰资源及其应用价值的研究). Resour Sci 2000, 22(6):22-26

40. He J, Shang Y: A high quality forage grass, Puspalum dilatatum (优质牧 草-毛花雀稗). Agric Tech Serv 2003, 10:18.

41. Li Z, Hao Y: Nutrition constituents of Coix lacryma-jobi and its comprehensive utilization (薏茨营养成分及综合利用). Agric Sci Technol Equip 2012, 5:75-76.

42. Wang Z: Medical value of Coix lacryma-jobi (薏药的药用价值). Applicable Technol Rural Areas 2004, 12:56-57.

43. Cai J: Nutrition and processing of Eleocharis dulcis (蒙荠的营养保健和加 工利用). Food and Nutr China 2005, 2:40-42.

44. Liu B, Gao Y, Wu H: Identification and application of three species from Acorus (三种菖蒲的鉴别和应用). Lishizhen Med Materia Medica Res 2004, 15(8):496.

45. Commerce Ministry of the People's Republic of China, Institute of Botany of CAS: Flora of China Economic Plants. Beijing: Science Press; 1961 [中华人民 共和国商业部土产废品局, 中国科学院植研所, 中国经济植物志, 科学出 版社: 北京, 1961].

46. Jiangsu New Medicine College (Ed.): A Grand Dictionary of Chinese Medicinal Herbs. Shanghai: Science Technology Publishing Co; 1995 [江苏新医学院, 中药大辞典, 上海科学技术出版社: 上海, 1995].

47. Wang J, Zhan Z: Use of Spirodela polyrrhiza in different history phases (浮 萍历代应用探源). Pharm Clin Chin Materia Med 2011, 2(4):39-40.

48. Zhang Z: Spirodela polyrrhiza, a high quality natural bait for grass carp (草鱼种的优质天然饵料——浮萍). Fishery Guide to be Rich 2000, 12:24.

49. Guo F: Murdannia triquetra, a high production and quality feed (高产优 质青饲料一水竹叶). Feed Res 1985, 2:31.

50. Zhao N, Xia G, Xu H, Du J, Pan L: Ethno-medicinal plants from Polygonum in Guizhou (贵州少数民族常用的蓼属植物药). J Med Pharm Chin Minorities 2012, 18(7):31-33.

51. Zhai S, Fu W, Xue M: Application of Polygonum hydropiper in study of pesticide and veterinary (水苶在农药和兽药研究中的应用). J Tradit Chin Vet Med 2008, 27(4):72-73.

52. Lu X: Using Polygonum hydropiper to cure diseases of livestock and poultry (用水䓹治疗畜禽疾病). Yunnan J Anim Sci Vet Med 2002, 1:46.

53. Li S: Leaves of Polygonum hydropiper can be used to treat metrorrhagia (水苶叶治子宫出血). Chin J Ethnomed Ethnopharmacy 2001, 3:157.

54. Wang B: Introduction to an aquatic plant, Nuphar pumila (水生植物萍蓬 草). Garden 2012, 4:70-71.

55. Wang $Y$, Wang $Q$, Wang $Y$ : Summery of medicinal plants from Polygonum in China (中国蓼属药用植物综述). ShiZhen J Tradit Chin Med Res 1996, 7(3):172-173.

56. Fang L, Liu H, Cui J, Xu Z: Traditional use of wetland plants in Dai villages in Xishuangbanna, Yunnan (西双版纳傣族村寨对湿地植物的传统利用). Biodivers Sci 2006, 14(4):300-308.

57. Chen M, Jia S: Flora of China Forage Plants. Beijing: China Agriculture Press; 1987 [陈默君，贾慎修，中国饲用植物志，中国农业出版社：北京, 1987]

58. Editing Group of Flora of Fujian: Flora of Fujian. Fuzhou: Fujian Science \& Technology Publishing House; 1982 [福建植物志编写组, 福建植物志, 福 建科学技术出版社; 福州, 1982].

59. Teaching and Research Group of Pharmacognosy, Second Military Medical University: An Illustrated Handbook of Medicinal Plants in China. Shanghai: Shanghai Education Press; 1960 [第二军医大学药学系生药学教研组, 中 国药用植物图鉴, 上海教育出版社; 上海, 1960].
60. Li B: New resources from Utricularia aurea for Aquariums (鱼缸水草新秀 一狸藻). Fisheries Sci Technol Inf 2001, 28(2):87-88.

61. Jing X: Stachys adulterina, a medicinal vegetable (药用蔬菜一草石蚕) Shanghai Vegetables 2004, 5:75.

62. Chen M, Jia S: Forage Plants in China. Beijing: China Agriculture Press; 2002 [陈默君，贾慎修，中国饲用植物，中国农业出版社：北京，2002]

63. Gui L, Li J, Tian F, Yang C, Liang S, Tian H: Protection and utilization of Guangxi wetland medicinal plant resources (广西湿地药用植物资源的 保护与利用). Contemp Med Forum 2014, 12(1):24-25.

64. Chu J: The investigation on the resources of aquatic plants in the west of Jilin province (吉林西部水生植物资源的调查). J Baicheng Normal Coll 2006, 20(4):1-4.

65. Deng F, Sun P, Li Q, Wu G, Deng F: Study on application and wastewater purification values of wetland aquatic plants (湿地水生植物的利用途径 与争化污水作用研究). Ecol Economy 2005, 4:66-69.

66. Yang Y, Zhang F, Zhang E, Dong C, A X, Tang C, Xu F, Dai L: The traditional use of agricultural plant resources by the Bulang people in Xishuangbanna, Yunnan province (云南西双版纳布朗族对农业植物的传 统利用研究). Chin Agric Sci Bull 2012, 28(33):177-186.

67. Zhang L, Song B, Wang R, Zhang F, Shangguang T: A study on the wild plant resources of wetlands in Shanxi (山西湿地野生植物资源研究). J Shanxi Univ (Nat Sci Ed) 1998, 21(3):281-285.

68. Shen S, Qian J, Ren J: Ethnoveterinary plant remedies used by Nu people in NW Yunnan of China. J Ethnobiol Ethnomed 2010, 6(1):24.

69. Ju Y, Zhuo J, Liu B, Long C: Eating from the wild: diversity of wild edible plants used by Tibetans in Shangri-la region, Yunnan, China. J Ethnobiol Ethnomed 2013, 9(1):28.

70. Wujisguleng W, Khasbagen K: An integrated assessment of wild vegetable resources in Inner Mongolian Autonomous Region, China. J Ethnobiol Ethnomed 2010, 6(1):34.

71. Li F, Li J, Liu B, Zhou J, Long C: Seeds used for Bodhi beads in China. $J$ Ethnobiol Ethnomed 2014, 10(1):15.

72. Ghorbani A, Langenberger G, Sauerborn J: A comparison of the wild food plant use knowledge of ethnic minorities in Naban River Watershed National Nature Reserve, Yunnan, SW China. J Ethnobiol Ethnomed 2012, 8(1):17.

73. Hodge WH: Chinese water chestnut or matai-a paddy crop of China. Econ Bot 1956, 10(1):49-65.

74. Huang J, Pei S, Long C: An ethnobotanical study of medicinal plants used by the Lisu people in Nujiang, northwest Yunnan, China. Econ Bot 2004, 58(1):S253-S264

75. Huai HY, Pei SJ: Plants used medicinally by folk healers of the Lahu people from the autonomous county of Jinping Miao, Yao, and Dai in southwest China. Econ Bot 2004, 58(1):S265-S273.

76. Keng H: Economic plants of ancient north China as mentioned in Shih Ching (Book of Poetry). Econ Bot 1973, 28(4):391-410.

77. Fu Y, Guo H, Chen A, Cui J, Padoch C: Relocating plants from swidden fallows to gardens in southwestern China. Econ Bot 2003, 57(3):389-402.

78. Au DT, Wu J, Jiang Z, Chen H, Lu G, Zhao Z: Ethnobotanical study of medicinal plants used by Hakka in Guangdong, China. J Ethnopharmacol 2008, 117(1):41-50,

79. Shang X, Tao C, Miao X, Wang D, Tangmuke D, Wang Y, Yang Y, Pan H: Ethno-veterinary survey of medicinal plants in Ruoergai region, Sichuan province, China. J Ethnopharmacol 2012, 142(2):390-400.

80. Khuankaew S, Srithi K, Tiansawat P, Jampeetong A, Inta A Wangpakapattanawong P: Ethnobotanical study of medicinal plants used by Tai Yai in Northern Thailand. J Ethnopharmacol 2014, 151(2):829-838.

81. Kang $Y$, Łuczaj $Ł$, Kang J, Zhang S: Wild food plants and wild edible fungi in two valleys of the Qinling Mountains (Shaanxi, central China). J Ethnobiol Ethnomed 2013, 9(1):26.

82. Kang Y, Łuczaj $Ł$, Kang J, Wang F, Hou J, Guo Q: Wild food plants used by the Tibetans of Gongba Valley (Zhouqu county, Gansu, China). J Ethnobiol Ethnomed 2014, 10(1):20.

83. Phillips O, Gentry AH: The useful plants of Tambopata, Peru: I. Statistical hypotheses tests with a new quantitative technique. Econ Bot 1993, 47(1):15-32.

84. Rossato SC, Leitão-Filho HDF, Begossi A: Ethnobotany of caiçaras of the Atlantic Forest coast (Brazil). Econ Bot 1999, 53(4):387-395.

85. Ladio AH, Lozada M: Nontimber forest product use in two human populations from northwest Patagonia: a quantitative approach. Hum Ecol 2001, 29(4):367-380.

86. Heinrich M, Ankli A, Frei B, Weimann C, Sticher O: Medicinal plants in Mexico: Healers' consensus and cultural importance. Soc Sci Med 1998 47(11):1859-1871. 
87. Hoffman B, Gallaher T: Importance Indices in Ethnobotany. Ethnobot Res Appl 2007, 5:201-218.

88. Naqinezhad A, Jalili A, Attar F, Ghahreman A, Wheeler BD, Hodgson JG, Shaw SC, Maassoumi A: Floristic characteristics of the wetland sites on dry southern slopes of the Alborz Mts., N. Iran: The role of altitude in floristic composition. Flora-Morphol Distrib Funct Ecol Plants 2009, 204(4):254-269.

89. Rasingam L: Aquatic and wetland plants of little andaman island, India. J Basic Appl Biol 2010, 4(3):52-59.

90. Saharia S, Sarma CM: Ethno-medicinal studies on indigenous wetland plants in the tea garden tribes of Darrang and Udalguri district, Assam, India. NeBIO 2011, 2:27-33.

91. Fu W, Li P, Bian $X$, Wu Y: Dynamics in the succession of vegetation communities in Inner-river wetland in Zhenjiang. Res Environ Yangtze Basin 2007, 16(2):163-168.

92. Lou Y, Zhao K, Ma K: Change in floristic composition and species diversity of plant community along environment gradient in Honghe National Nature Reserve, China. Acta Ecol Sin 2007, 27(9):3843-3891.

93. Wu Q, Xia X, Ye J, Jiang L, Miu K, Ding B: Plant diversity and ecosystem health assessment of Sanyang wetland in Wenzhou, Zhejiang. J Zhejiang Univ (Agric Life Sci) 2012, 38(4):421-428.

94. Sun R, Zhang L, Wang T, Chen F, Deng Q: Research on characteristics and species diversity of plant community of different kinds of wetlands in Hefei. Chin Wild Plant Resour 2013, 32(4):34-40.

95. Cheng L, Chen K, Wang S, Yang S, Su X, Wang J: Plant Diversity of Xiaopohu Wetlands in Qinghai Lake Basin. Wetl Sci 2013, 11(4):460-465.

96. Jain A, Roshnibala S, Kanjilal PB, Singh RS, Singh HB: Aquatic/semi-aquatic plants used in herbal remedies in the wetlands of Manipur, Northeastern India. Indian J Tradit Know 2007, 6(2):346-351.

97. Moerman DE, Pemberton RW, Kiefer D, Berlin B: A comparative analysis of five medicinal floras. J Ethnobio 1999, 19(1):49-67.

98. Siwakoti M: An overview of floral diversity in wetlands of Terai region of Nepal. Our Nature 2006, 4(1):83-90.

99. Boro A, Sarma GC: Ethnic uses of some wetland plants by the Bodo community in Udalgiri district of Assam, India. Pleione 2013, 7(1):155-159.

100. Levetin E, McMahon K: Plant \& Society. 5th edition. New York: Mcgraw-Hill; 2008.

101. Hoft M, Barik SK, Lykke AM: Quantitative ethnobotany: Applications of multivariate and statistical analyses in ethnobotany. UNESCO, People and Plants working, paper Paris; 1999.

102. Hasbagan HH: Quantitative methods in Ethnobotany (II). J Inner Mongolia Normal Univ (Nat Sci Ed) 2010, 39(4):417-419.

103. Heinrich M: Ethnobotany and its role in drug development. Phytother Res 2000, 14(7):479-488

104. Rokaya MB, Münzbergová Z, Timsina B: Ethnobotanical study of medicinal plants from the Humla district of western Nepal. J Ethnopharmacol 2010, 130(3):485-504

105. Ayyanar M, Ignacimuthu S: Ethnobotanical survey of medicinal plants commonly used by Kani tribals in Tirunelveli hills of Western Ghats, India. J Ethnopharmacol 2011, 134(3):851-864.

106. Cadena-González AL, Sørensen M, Theilade I: Use and valuation of native and introduced medicinal plant species in Campo Hermoso and Zetaquira, Boyacá, Colombia. J Ethnobiol Ethnomed 2013, 9(1):23.

107. Vitalini S, Iriti M, Puricelli C, Ciuchi D, Segale A, Fico G: Traditional knowledge on medicinal and food plants used in Val San Giacomo (Sondrio, Italy) - An alpine ethnobotanical study. J Ethnopharmacol 2013, 145(2):517-529.

108. Beltran L, Ortiz A, Mariano N, Maldonado B, Reyes V: Factors affecting ethnobotanical knowledge in a mestizo community of the Sierra de Huautla Biosphere Reserve, Mexico. J Ethnobiol Ethnomed 2014, 10(1):14.

109. Huai H, Dong Q, Liu A: Ethnomedicinal analysis of toxic plants from five ethnic groups in China. Ethnobot Res Appl 2010, 8:169-179.

110. Clevering OA, Lissner J: Taxonomy, chromosome numbers, clonal diversity and population dynamics of Phragmites australis. Aquat Bot 1999, 64(3):185-208.

111. Saltonstall K: Cryptic invasion by a non-native genotype of the common reed, Phragmites australis, into North America. Proc Natl Acad Sci U S A 2002, 99(4):2445-2449.

doi:10.1186/1746-4269-10-72

Cite this article as: Zhang et al:: Diversity of wetland plants used traditionally in China: a literature review. Journal of Ethnobiology and Ethnomedicine 2014 10:72.

\section{Submit your next manuscript to BioMed Central and take full advantage of:}

- Convenient online submission

- Thorough peer review

- No space constraints or color figure charges

- Immediate publication on acceptance

- Inclusion in PubMed, CAS, Scopus and Google Scholar

- Research which is freely available for redistribution 